\title{
Combining the GW formalism with the polarizable continuum model: A state-specific non-equilibrium approach
}

Ivan Duchemin, Denis Jacquemin, and Xavier Blase

Citation: The Journal of Chemical Physics 144, 164106 (2016);

View online: https://doi.org/10.1063/1.4946778

View Table of Contents: http://aip.scitation.org/toc/jcp/144/16

Published by the American Institute of Physics

\section{Articles you may be interested in}

Bethe-Salpeter study of cationic dyes: Comparisons with ADC(2) and TD-DFT

The Journal of Chemical Physics 146, 034301 (2017); 10.1063/1.4974097

GW and Bethe-Salpeter study of small water clusters

The Journal of Chemical Physics 144, 034109 (2016); 10.1063/1.4940139

The energy level alignment at metal-molecule interfaces using Wannier-Koopmans method Applied Physics Letters 108, 262104 (2016); 10.1063/1.4955128

Excited-state potential-energy surfaces of metal-adsorbed organic molecules from linear expansion $\Delta$-selfconsistent field density-functional theory $(\triangle \mathrm{SCF}-\mathrm{DFT})$

The Journal of Chemical Physics 139, 014708 (2013); 10.1063/1.4812398

A state-specific polarizable continuum model time dependent density functional theory method for excited state calculations in solution

The Journal of Chemical Physics 125, 054103 (2006); 10.1063/1.2222364

Electronic excitation energies of molecules in solution within continuum solvation models: Investigating the discrepancy between state-specific and linear-response methods

The Journal of Chemical Physics 123, 134512 (2005); 10.1063/1.2039077

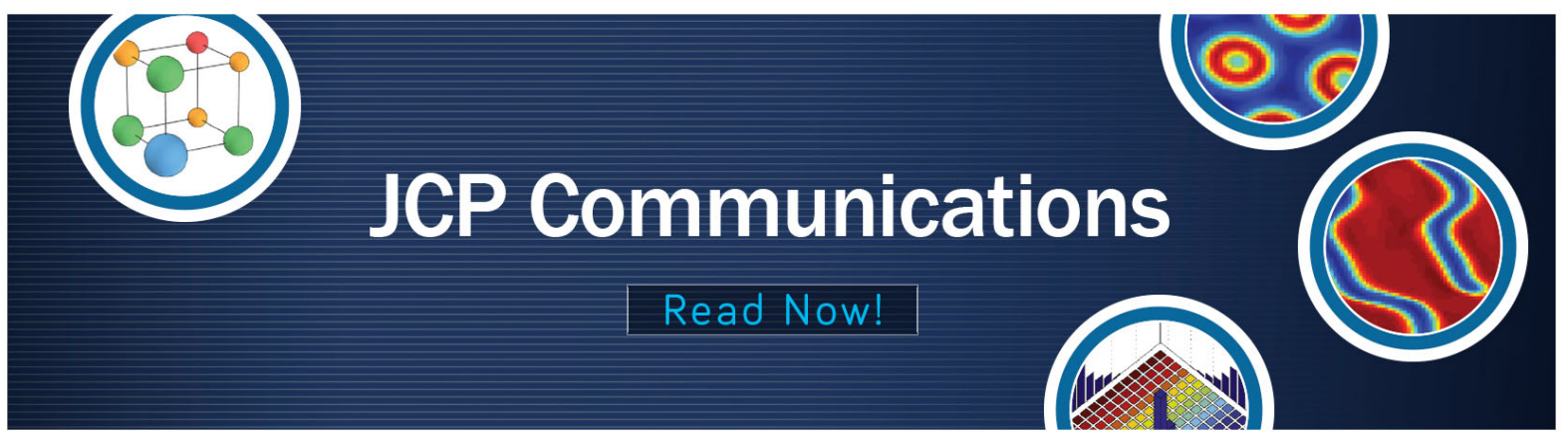




\title{
Combining the GW formalism with the polarizable continuum model: A state-specific non-equilibrium approach
}

\author{
Ivan Duchemin, ${ }^{1, a)}$ Denis Jacquemin, ${ }^{2,3}$ and Xavier Blase ${ }^{4,5}$ \\ ${ }^{1} I N A C$, SP2M/L_Sim, CEA/UJF Cedex 09, 38054 Grenoble, France \\ ${ }^{2}$ Laboratoire CEISAM - UMR CNR 6230, Université de Nantes, 2 Rue de la Houssinière, \\ BP 92208, 44322 Nantes Cedex 3, France \\ ${ }^{3}$ Institut Universitaire de France, 1 rue Descartes, 75005 Paris Cedex 5, France \\ ${ }^{4}$ CNRS, Inst. NÉEL, F-38000 Grenoble, France \\ ${ }^{5}$ Univ. Grenoble Alpes, Inst. NÉEL, F-38000 Grenoble, France
}

(Received 17 February 2016; accepted 1 April 2016; published online 25 April 2016)

\begin{abstract}
We have implemented the polarizable continuum model within the framework of the many-body Green's function $G W$ formalism for the calculation of electron addition and removal energies in solution. The present formalism includes both ground-state and non-equilibrium polarization effects. In addition, the polarization energies are state-specific, allowing to obtain the bath-induced renormalisation energy of all occupied and virtual energy levels. Our implementation is validated by comparisons with $\triangle \mathrm{SCF}$ calculations performed at both the density functional theory and coupledcluster single and double levels for solvated nucleobases. The present study opens the way to $G W$ and Bethe-Salpeter calculations in disordered condensed phases of interest in organic optoelectronics, wet chemistry, and biology. Published by AIP Publishing. [http://dx.doi.org/10.1063/1.4946778]
\end{abstract}

\section{INTRODUCTION}

The study of electronic excitations in complex disordered systems remains a challenge for computational methods, leading to various strategies to split the system into an "active" portion of space, where the highest desired level of Quantum Mechanical (QM) treatment is performed, and an "environmental" surrounding medium (a solvent, a metallic nanoparticle, a disordered polymeric phase, etc.) that is considered at a lower level of theory. This lower level can be either a simplified $\mathrm{QM}$ model (QM/QM'), a molecular mechanical approach (QM/MM), or a continuum model representing the environment as a structureless material having realistic macroscopic properties. This latter partitioning allows in particular to straightforwardly account for the renormalization of the excitation energy in the QM part due to the re-arrangement of charges in the polarizable environmental part induced by the excitation inside the QM region. The description of the dielectric response of the surrounding polarizable medium can notably be achieved with the Polarizable Continuum Model (PCM) ${ }^{1}$ or within a discrete model with, e.g., induced charges and/or dipoles at atomic sites.

Since its first introduction in $1981,{ }^{2}$ much progress has been achieved along the line of the PCM approach where the QM part (the solute) is considered to be located inside a cavity carved into a continuous polarizable material (representing, e.g., the solvent) characterized by its macroscopic dielectric constant $(\epsilon)$. The definition of the cavity and the discretization of its surface, ${ }^{3,4}$ the scheme used to extract the apparent surface or volume charges that will enforce the electrostatic field and potential continuity equations across the boundary between

\footnotetext{
a)Electronic mail: ivan.duchemin@cea.fr
}

the two media, from the original D-PCM ${ }^{2}$ to the IEF-PCM ${ }^{5,6}$ or the $\mathrm{SS}(\mathrm{V}) \mathrm{PE}^{7,8}$ formulations, the treatment of anisotropic dielectric properties in the continuum, ${ }^{5}$ the inclusion of nonelectrostatic contributions to the solvation energy, $, 9,10$ and the different strategies adopted to minimize the problems associated with the "leaking" of the QM density outside the cavity, ${ }^{5,8}$ are important issues that have been worked out by the community over the years. Further, beyond the exact treatment of the boundary conditions, approximate but efficient schemes, such as the conductor-like-screening-model (COSMO) approach, ${ }^{11}$ allowing the explicit calculation of solvation energies and gradients, can be used as an alternative to the IEF-PCM or SS(V)PE schemes. Likewise, we mention that multipolar representation of the solvent was also proposed as early as $1973 .{ }^{12,13}$ A review of the pros and cons of all these approaches is beyond the scope of the present study and we refer the reader to recent reviews where in-depth discussions are provided. ${ }^{14-16}$

Concerning the quantum-mechanical approach used to describe the solute, we consider here a specific formulation of many-body perturbation theory, labeled the $G W$ formalism, ${ }^{17-25}$ that has recently attracted a growing interest for the study of isolated molecular systems. ${ }^{26-54}$ Such a formalism relies on an approximation to the equation-ofmotion of the time-ordered one-body Green's function $(G)$ of which the poles are the addition and removal energies, namely the difference of energies between the eigenstates of the $(N \pm 1)$-electrons systems and the $N$-electron ground state. As such, the poles of the Green's function are the correct vertical excitation energies upon addition of an integer charge (electron or hole) to the $N$-electron system, with the advantages as compared to standard $\triangle \mathrm{SCF}$ calculations that: (a) all occupied and virtual energy levels can be straightforwardly obtained, not only the frontier orbital energies; and (b) the formalism 
can be used for both finite or extended systems. The main limitation of the $G W$ approach is computational cost, with a $O\left(N^{4}\right)$ scaling, but systems containing well above a hundred atoms can now be routinely studied. ${ }^{36,45,51,55,56}$ Concerning the obtained accuracy, existing studies and benchmarks on gas phase molecules or complexes have demonstrated that errors limited to a very few tenths of an $\mathrm{eV}$ can be obtained as compared to experimental or higher-level calculations such as $\operatorname{CCSD(T).~This~will~be~illustrated~below~in~the~case~}$ of nucleobases. Finally, the $G W$ approach opens the way to the study of optical excitations within the framework of the Bethe-Salpeter equation (BSE) formalism ${ }^{57}$ that has been shown recently, thanks to thorough benchmarks on several molecular families, ${ }^{58-60}$ to provide average accuracies comparable with the best ("optimized") time-dependent density functional theory (TD-DFT) approaches, solving in particular the standard problems associated with chargetransfer excitations ${ }^{36,47,61-64}$ and cyanine-like ${ }^{65}$ systems.

Contrary to, e.g., density functional theory (DFT) and its time-dependent (TD-DFT) extension, the merging of the $G W$ formalism with polarizable models has been very scarcely addressed by the community, reflecting the fact that such a technique has been originally developed since the mid-1960s for periodic extended systems. In a pioneering study, Rohlfing introduced model dielectric functions to account for the long-range screening by a substrate in the so-called $L D A+G d W$ approach. ${ }^{66}$ As another direction of progress, $G W$ and BSE calculations were recently achieved starting from eigenstates generated with a QM/MM approach at the DFT level, namely ground-state polarization effects were introduced, but the excitations out of the groundstate were not screened. ${ }^{67,68}$ Finally, a polarizable model with a regular grid of polarizable centres was recently combined with the $G W$ and BSE formalisms for the study of Frenkel and charge-transfer excitations in a donor/acceptor complex. $^{45}$

In the present study, we explore the merits of combining the $G W$ formalism with the polarizable continuum model. Our goal is not to explore the merits of the PCM, but to validate its combination with the $G W$ formalism since several approximations are required for merging the two formalisms. Such approximations are validated quantitatively in the case of hydrated nucleobases. The choice of such systems is dictated by the existence of several reference calculations at various quantum mechanical levels and experimental data. Further, the large difference between the static and optical dielectric constant of water $\left(\epsilon \sim 78.35\right.$ and $\epsilon_{\infty} \sim 1.78$, respectively) allows to properly introduce the importance of the socalled non-equilibrium PCM effects that can be naturally introduced in the $G W$ framework. We show in particular that the solvent induced shifts obtained at the $(G W+\mathrm{PCM})$ level are within a few percent of that obtained by $(\triangle \mathrm{SCF}+\mathrm{PCM})$ techniques at the DFT or CCSD levels, as implemented within a reference computational package, namely Gaussian09. ${ }^{69}$ However, contrary to standard $\triangle \mathrm{SCF}$ calculations that provide the energy renormalisation of frontier orbitals, we show that the $G W$ formalism allows to obtain directly the solvent-induced shift of the entire occupied and virtual spectrum.
The present study is organized as follows. We briefly expose in Section II the $G W$ formalism, emphasizing the issues of importance concerning the combination with the PCM model. In particular, the so-called static Coulomb-hole plus screened-exchange (COHSEX) limit for the $G W$ operator, and the contribution of the continuous dielectric medium to the screened Coulomb potential $W$ inside the quantum mechanical cavity, are emphasized as crucial ingredients for the present implementation. Our results are presented in Section III followed by a discussion in Section IV. We leave to the Appendix a discussion of our implementation of the PCM model within a modified IEF-PCM implementation accounting explicitly for the leaking of the charge outside of the cavity.

\section{THE GW FORMALISM AND THE DYNAMICALLY SCREENED COULOMB POTENTIAL $W$}

\section{A. Basic equations}

We briefly review the $G W$ formalism, emphasizing the features of relevance for the embedding strategy we develop. More details can be found in thorough reviews on the subject. ${ }^{17-25}$ We start by introducing the time-ordered Green's function $G$ that reads

$$
\begin{aligned}
i \hbar G\left(\mathbf{r} t, \mathbf{r}^{\prime} t^{\prime}\right)= & \theta\left(t-t^{\prime}\right)\left\langle\psi_{G S}(N)\left|\hat{\psi}\left(\mathbf{r}^{\prime} t^{\prime}\right) \hat{\psi}^{\dagger}(\mathbf{r} t)\right| \psi_{G S}(N)\right\rangle \\
& -\theta\left(t^{\prime}-t\right)\left\langle\psi_{G S}(N)\left|\hat{\psi}^{\dagger}(\mathbf{r} t) \hat{\psi}\left(\mathbf{r}^{\prime} t^{\prime}\right)\right| \psi_{G S}(N)\right\rangle,
\end{aligned}
$$

where $\psi_{G S}(N)$ is the $N$-electron ground-state wavefunction and $\left\{\hat{\psi}(\mathbf{r} t), \hat{\psi}^{\dagger}(\mathbf{r} t)\right\}$ are the destruction/creation field-operators in the Heisenberg representation. As such, $G$ follows the propagation in time of an added (removed) electron to (from) the $N$-electron system in its ground-state. It can be shown in consequence that its spectral representation (after time to frequency Fourier transform) is dominated by energy poles that are precisely the addition (removal) energies of an electron to (from) the $N$-electron ground-state, including the ionization energy and electronic affinity. The so-called Lehman representation of $G$ reads

$$
G\left(\mathbf{r}, \mathbf{r}^{\prime} ; \omega\right)=\sum_{a} \frac{g_{a}(\mathbf{r}) g_{a}^{*}\left(\mathbf{r}^{\prime}\right)}{\omega-E_{a}+i 0^{+}}+\sum_{i} \frac{g_{i}(\mathbf{r}) g_{i}^{*}\left(\mathbf{r}^{\prime}\right)}{\omega-E_{i}-i 0^{+}},
$$

where $E_{a}=E_{a}(N+1)-E_{0}(N)$ is an addition energy, with the (a) indexing the eigenstates of the $(N+1)$-electron system, while $E_{i}=E_{0}(N)-E_{i}(N-1)$ span the removal energies. The $g_{a / i}$ are called Lehman amplitudes and the infinitesimally small $\left(0^{+}\right)$positive parameter indicates that the Green's function can only be analytically continued in the first and third quadrants of the $\omega$-frequency complex plane. ${ }^{24,70}$

Anticipating on the comparisons that will be conducted below, it is interesting to consider the standard $\triangle \mathrm{SCF}$ techniques that proceed by calculating the ground-state energy of the $(N+1)$, neutral, and $(N-1)$-electron systems. Both the $G W$ and $\triangle \mathrm{SCF}$ formalisms are thus concerned with the explicit change in the number of electrons in the system. In the $\triangle \mathrm{SCF}$ approach, the electronic reorganization energy associated with the change in particle number is usually treated 
self-consistently, e.g., at the density functional level. In the case of the $G W$ formalism, the response of the $N$-electron system is contained in the dynamical susceptibility $P\left(\mathbf{r}, \mathbf{r}^{\prime} ; \omega\right)$ that relates the self-consistent changes in charge density to the perturbation that represents the added electron or hole. The screened Coulomb-potential $W$ that enters the $G W$ formalism is closely related to the susceptibility operator (see below).

On very general grounds, the equation-of-motion of the Green's function leads to the following eigenvalue equation where we introduce the self-energy operator $\Sigma$ that represents exchange and correlation effects:

$$
\begin{aligned}
& {\left[\omega-h_{0}(\mathbf{r})\right] G\left(\mathbf{r}, \mathbf{r}_{0} ; \omega\right)} \\
& \quad-\int d \mathbf{r}^{\prime} \Sigma\left(\mathbf{r}, \mathbf{r}^{\prime} ; \omega\right) G\left(\mathbf{r}^{\prime}, \mathbf{r}_{0} ; \omega\right)=\delta\left(\mathbf{r}-\mathbf{r}_{0}\right),
\end{aligned}
$$

where the one-body Hamiltonian $h_{0}$ contains the kinetic energy, ionic, and Hartree operators. Within the $G W$ formalism, which can be considered as the lowest-order approximation to $\Sigma$ in terms of the screened Coulomb potential $W$, the quantities to be calculated read

$$
\begin{aligned}
\Sigma\left(\mathbf{r}, \mathbf{r}^{\prime} ; E\right) & =\frac{i}{2 \pi} \int d \omega e^{i \omega 0^{+}} G\left(\mathbf{r}, \mathbf{r}^{\prime} ; E+\omega\right) W\left(\mathbf{r}, \mathbf{r}^{\prime} ; \omega\right), \\
G\left(\mathbf{r}, \mathbf{r}^{\prime} ; E\right) & =\sum_{n} \frac{\phi_{n}(\mathbf{r}) \phi_{n}^{*}\left(\mathbf{r}^{\prime}\right)}{E-\varepsilon_{n}+i 0^{+} \times \operatorname{sgn}\left(\varepsilon_{n}-E_{F}\right)}, \\
W\left(\mathbf{r}, \mathbf{r}^{\prime} ; \omega\right) & =v\left(\mathbf{r}, \mathbf{r}^{\prime}\right)+\int d \mathbf{r}_{1} d \mathbf{r}_{2} v\left(\mathbf{r}, \mathbf{r}_{1}\right) P_{0}\left(\mathbf{r}_{1}, \mathbf{r}_{2} ; \omega\right) W\left(\mathbf{r}_{2}, \mathbf{r}^{\prime} ; \omega\right), \\
P_{0}\left(\mathbf{r}, \mathbf{r}^{\prime} ; \omega\right) & =\sum_{i, j}\left(f_{i}-f_{j}\right) \frac{\phi_{i}^{*}(\mathbf{r}) \phi_{j}(\mathbf{r}) \phi_{j}^{*}\left(\mathbf{r}^{\prime}\right) \phi_{i}\left(\mathbf{r}^{\prime}\right)}{\varepsilon_{i}-\varepsilon_{j}-\omega-i 0^{+} \times \operatorname{sgn}\left(\varepsilon_{i}-\varepsilon_{j}\right)},
\end{aligned}
$$

where $v\left(\mathbf{r}, \mathbf{r}^{\prime}\right)$ is the bare Coulomb potential, $G$ the timeordered one-body Green's function, $P_{0}$ the independentelectron susceptibility, and $W$ the screened Coulomb potential. The $\left\{f_{i / j}\right\}$ are occupation numbers and the input $\left\{\phi_{n}, \varepsilon_{n}\right\}$ eigenstates are typically Kohn-Sham eigenstates that will be corrected within the present $G W$ formalism. Once the selfenergy $\Sigma$ is obtained, inserting the Lehman representation for $G$, one obtains

$$
h_{0}(\mathbf{r}) \psi_{n}(\mathbf{r})+\int d \mathbf{r}^{\prime} \Sigma\left(\mathbf{r}, \mathbf{r}^{\prime} ; E_{n}\right) \psi_{n}\left(\mathbf{r}^{\prime}\right)=E_{n} \psi_{n}(\mathbf{r}),
$$

where the $\left\{g_{n}\right\}$ Lehman amplitudes have been renamed $\left\{\psi_{n}\right\}$ to bridge with more standard notations for the one-body eigenvector equation. Note that the $\left(E_{n}, \psi_{n}\right)$ solutions of Eq. (7) can be different from the $\left(\varepsilon_{n}, \phi_{n}\right)$ states used to build $W$ and $\Sigma$, e.g., input Kohn-Sham eigenstates. This equation generalizes the standard Kohn-Sham or Hartree-Fock eigenvalue problem since $\Sigma$ is energy-dependent. In practice, the standard and most simple $G W$ approach consists of replacing the DFT exchangecorrelation potential contribution to the input Kohn-Sham eigenvalues by the $G W$ self-energy operator,

$$
E_{n}^{G W}=\varepsilon_{n}^{K S}+\left\langle\phi_{n}^{K S}\left|\Sigma^{G W}\left(E_{n}^{G W}\right)-V^{X C}\right| \phi_{n}^{K S}\right\rangle .
$$

With the self-energy operator built from the input Kohn-Sham DFT eigenstates, such a simple non-self-consistent scheme is labeled as the $G_{0} W_{0}$ approach. More specifically, if the starting DFT calculation is performed with, e.g., the PBE0 exchangecorrelation functional, ${ }^{71}$ the notation $G_{0} W_{0} @$ PBE0 is used. As documented in several recent studies, such an approach leads for organic molecules to ionization potentials too small with respect to experiment when starting with semilocal DFT functionals or hybrids with a relatively limited amount of exact exchange such as PBE0. As illustrated here below, better energies can be obtained by using a simple self-consistent scheme where the corrected eigenvalues are reinjected into the construction of $G$ and $P_{0}$ in Eqs. (4) and (6). Such a scheme, labeled ev $G W$ in the following, is a partially self-consistent approach with update of the eigenvalues only. We refer the readers to recent papers devoted to self-consistency within $G W$ for molecular systems. ${ }^{30,31,37-39,41,44,46,48,49,54}$

\section{B. PCM contribution to the screened-Coulomb potential $\mathbf{W}$}

Observing the definition, in Eq. (6), of the independentelectron polarizability $P_{0}$ built with occupied/virtual KohnSham eigenstates, it is obvious that if two systems located in two separate regions (1) and (2) have non-overlapping molecular orbitals, there are no matrix elements $P_{0}\left(\mathbf{r}_{1}, \mathbf{r}_{2}\right)$ coupling 1 and 2 . Using a block notation where index 1 (2) corresponds to points located in area 1 (2), the restriction $W_{11}$ of the screened Coulomb potential to system 1 reads

$$
\begin{aligned}
& W_{11}=v_{11}+v_{11} P_{0,11} W_{11}+v_{12} P_{0,22} W_{21}, \\
& W_{21}=v_{21}+v_{21} P_{0,11} W_{11}+v_{22} P_{0,22} W_{21} .
\end{aligned}
$$

Simple matrix manipulation leads to the following formulation for the restriction of the screened Coulomb potential to system (1):

$$
\begin{aligned}
W_{11} & =\tilde{v}_{11}+\tilde{v}_{11} P_{0,11} W_{11}, \\
\tilde{v}_{11} & =v_{11}+v_{12} P_{22}^{*} v_{21}, \\
P_{22}^{*} & =P_{0,22}+P_{0,22} v_{22} P_{22}^{*} .
\end{aligned}
$$

The quantity $P_{22}^{*}$ is the interacting polarizability of system (2) alone, namely in the absence of system 1. We have introduced the $(*)$ subscript to indicate that it should not be 
confused with the restriction to system (2) of the interacting polarizability of the full $(1+2)$ system. Since the interacting polarizability relates the variation of the charge density as a response to an applied perturbation, the interpretation of the contribution of $v_{12} P_{22}^{*} v_{21}$ to $\tilde{v}_{11}$ is straightforward. Reintroducing explicit real-space indexes, the quantity

$$
\Delta n\left(\mathbf{r}_{2}\right)=\int d \mathbf{r}_{2}^{\prime} P^{*}\left(\mathbf{r}_{2}, \mathbf{r}_{2}^{\prime}\right) v\left(\left|\mathbf{r}_{2}^{\prime}-\mathbf{r}_{1}\right|\right)
$$

represents the charge density variation generated in $\left(\mathbf{r}_{2}\right)$ inside system (2) by a unit test charge located in $\left(\mathbf{r}_{1}\right)$ inside the cavity. As a result, the term

$V^{\mathrm{reac}}\left(\mathbf{r}_{1}, \mathbf{r}_{1}^{\prime}\right)=\int d \mathbf{r}_{2} d \mathbf{r}_{2}^{\prime} v\left(\left|\mathbf{r}_{1}^{\prime}-\mathbf{r}_{2}\right|\right) P^{*}\left(\mathbf{r}_{2}, \mathbf{r}_{2}^{\prime}\right) v\left(\left|\mathbf{r}_{2}^{\prime}-\mathbf{r}_{1}\right|\right)$

is the reaction potential generated on point $\mathbf{r}_{1}^{\prime}$ in system (1) by the charge variation $\Delta n\left(\mathbf{r}_{2}\right)$ in system (2). As such, the renormalized Coulomb interaction $\tilde{v}_{11}\left(\mathbf{r}_{1}, \mathbf{r}_{1}^{\prime}\right)$ is just the sum of the bare Coulomb potential generated on $\mathbf{r}_{1}^{\prime}$ by a normalized test charge in $\mathbf{r}_{1}$, with $\mathbf{r}_{1}$ and $\mathbf{r}_{1}^{\prime}$ inside the cavity, plus the reaction field on $\mathbf{r}_{1}^{\prime}$ generated by the polarization charges developed in system (2) as a response to the test charge in $\mathbf{r}_{1}$ (see Fig. 1 for a symbolic representation). In this way, we recover the physics of solving Poisson's equation inside a cavity surrounded by a dielectric medium characterized by its dielectric response properties.

In the present Coulomb-fitting resolution-of-the-identity (RI-V) Gaussian basis implementation, ${ }^{72}$ we do not calculate $\tilde{v}_{11}\left(\mathbf{r}_{1}, \mathbf{r}_{1}^{\prime}\right)$, but the Gaussian integrals

$$
\tilde{v}_{\beta, \beta^{\prime}}=\int d \mathbf{r}_{1} d \mathbf{r}_{1}^{\prime} \beta\left(\mathbf{r}_{1}\right) \tilde{v}_{11}\left(\mathbf{r}_{1}, \mathbf{r}_{1}^{\prime}\right) \beta^{\prime}\left(\mathbf{r}_{1}^{\prime}\right)
$$

namely the two-centers two-electrons Coulomb integrals between "auxiliary" Gaussian orbitals located on the atomic sites in the QM region. In practice, this means that for the calculation of the $\tilde{v}_{\beta, \beta^{\prime}}$ matrix elements, we calculate the reaction field associated with a charge supported by an auxiliary basis $\beta$ orbital in the quantum mechanical part and

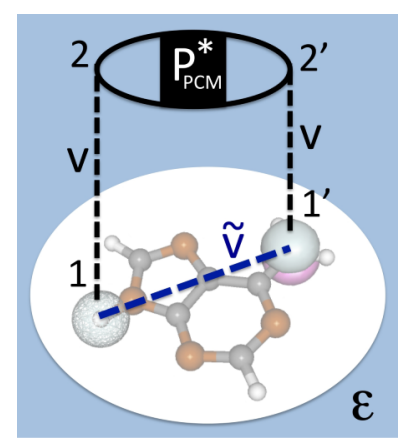

FIG. 1. Symbolic representation of the renormalization of the Coulomb integrals between auxiliary basis orbitals in the quantum mechanical part through polarization of the continuous polarizable medium. The symbols $v$ and $\tilde{v}$ stand for the bare and renormalized Coulomb interactions whereas $P_{\mathrm{PCM}}^{*}$ is the polarizability of the PCM in the absence of the solute. Embedded $G W$ calculations are performed as in the vacuum provided that the renormalized $\tilde{v}$ Coulomb potential is used when building the screened Coulomb potential $W$ (see Eq. (11) in the text). The shape of the cavity drawn here is symbolic and does not reflect the standard construction based on atom-centered spheres. calculate the action of the generated induced charges and dipoles onto all $\beta^{\prime}$ orbitals in the QM region. More details about the PCM implementation can be found in the Appendix. Once $\tilde{v}_{11}$ is built, one can proceed with a standard $G W$ calculation for the isolated quantum mechanical part provided that one uses $\tilde{v}$ in the construction of $W$. In particular, once $\tilde{v}_{\beta, \beta^{\prime}}$ is known, the effect of the PCM onto all occupied/virtual electronic energy levels can be obtained.

\section{From dynamical to static screening}

A difficulty to combine the $G W$ formalism with the PCM approach is that the calculation of the self-energy operator $\Sigma\left(\mathbf{r}, \mathbf{r}^{\prime} ; \omega\right)$ requires the knowledge of the dynamically screened (namely $\omega$-dependent) Coulomb potential $W\left(\mathbf{r}, \mathbf{r}^{\prime} ; \omega\right)$. The frequency dependence of $W$ stems from the frequencydependence of the dielectric function in the optical range. This is schematically represented in Fig. 2 where the real-part of the optical macroscopic dielectric constant of water, as defined from the frequency-dependent refractive index, is represented in the optical range and beyond. In other words, the relation connecting the screened Coulomb potential $W_{11}$ in the QM region to the polarizability $P_{0}$ in the QM and PCM subsystems is frequency dependent

$W_{11}(\omega)=v_{11}+v_{11} P_{0,11}(\omega) W_{11}(\omega)+v_{12} P_{0,22}(\omega) W_{21}(\omega)$.

While the explicit frequency dependence of the QM solute $P_{0,11}(\omega)$ polarizability is calculated explicitly from Eq. (6), the frequency-dependence of the polarizable medium optical dielectric constant is usually not available since standard PCM implementations only consider the $(\omega \rightarrow 0)$ static limit of the optical dielectric constant when it comes to screen

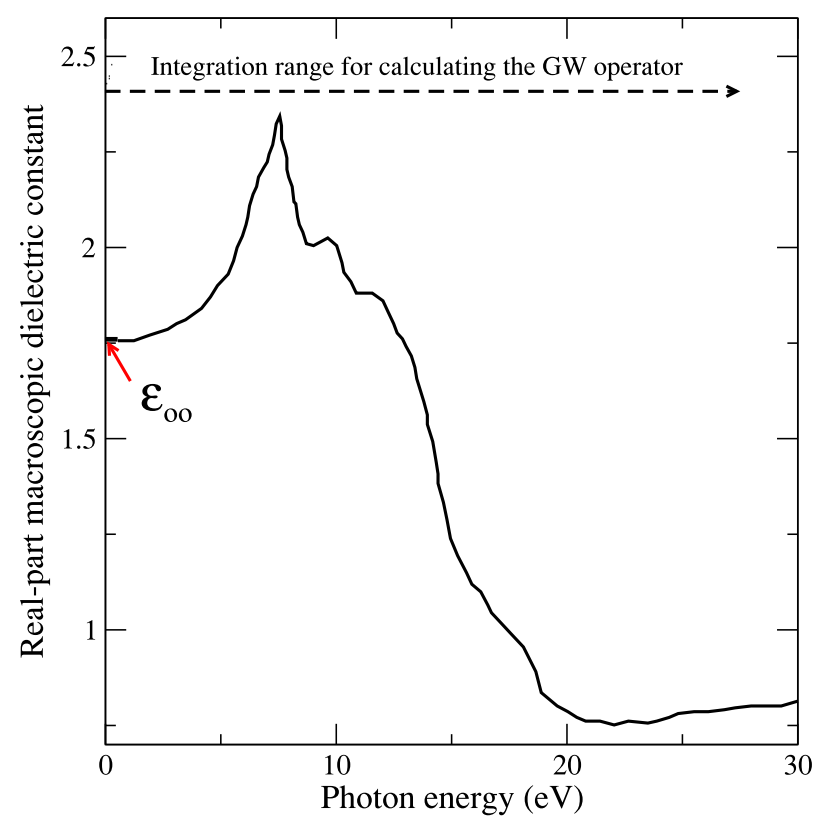

FIG. 2. Dependence of the optical dielectric constant of water on photon energy (adapted from experimental data of Ref. 73). The $G W$ self-energy operator requires the knowledge of the optical microscopic dielectric function $\epsilon\left(\mathbf{r}, \mathbf{r}^{\prime} ; \omega\right)$ for $0 \leq \omega<\infty$. The static COHSEX approximation only requires the knowledge of $\epsilon\left(\mathbf{r}, \mathbf{r}^{\prime} ; \omega=0\right)$ with the corresponding $\epsilon_{\infty}$ macroscopic dielectric constant value $\left(\epsilon_{\infty} \sim 1.78\right.$ for water; see text $)$. 
"fast" electronic excitations in a photoemission or optical experiment. This is usually labeled $\left(\epsilon_{\infty}\right)$ with for example $\epsilon_{\infty} \sim 1.78$ for water (see red arrow in Fig. 2). Even though $\epsilon_{\infty}$ is taken to be the static limit of the optical dielectric constant, related to the optical refractive index, it should not be confused with the "static dielectric constant," that is, $(\epsilon \simeq 78)$ for water, that accounts both for ionic and electronic degrees of freedom.

We will not explore the generalization of the PCM formalism to a full frequency-dependent dielectric response in the optical range. While certainly possible, we show below that simple schemes relying on the PCM implementations as they stand already lead to excellent results. As such, a first strategy consists in ignoring the above-mentioned difficulty and just assume that the dielectric constant of the polarizable medium is constant in the optical range. We therefore perform a $G W$ calculation in the gas phase and a $G W$ calculation in the presence of the PCM, replacing the bare Coulomb potential $v$ by its $\tilde{v}$ analog, where $\tilde{v}$ is renormalized but frequency independent. The change in $G W$ quasiparticle energies upon switching on the PCM directly provides the solvation energy shifts. Such a straightforward scheme will be labeled $\Delta G W$ in the following.

A second strategy, proposed, e.g., in the case of the renormalization by a metallic electrode of molecular frontier orbitals energy, ${ }^{74,75}$ consists in combining the polarizable medium response with a "low-frequency-screening-only" formulation of $G W$. Namely, rather than generalizing the PCM model to frequency-dependent screening properties, we restrict the $G W$ formalism to a frequency-independent screening approach. There exists indeed a well documented restriction of the $G W$ formalism to low-frequency $(\omega=0)$ screening, the so-called "static" Coulomb-hole plus screenedexchange (COHSEX) approximation that was introduced by Lars Hedin in his seminal paper introducing the $G W$ formalism. ${ }^{18}$ Again, the word "static" means here that only the $(\omega=0)$ low-frequency limit $\left(\epsilon_{\infty}\right)$ of the optical dielectric constant is considered. Such a COHSEX approximation was very largely used in the context of (simplified) self-consistent $G W$ approaches. ${ }^{76}$ While not providing quasiparticle energies as accurate as the $G W$ formalism, we show below that the solvatochromic shift, namely the difference between the quasiparticle energies provided by COHSEX calculations performed with and without PCM, reproduces very closely the shifts obtained using standard $\triangle \mathrm{SCF}$ DFT or CCSD calculations. In practice, we write

$$
\begin{aligned}
\left\{\Sigma^{G W}\right. & \left.+\operatorname{PCM}\left(\epsilon_{\infty}\right)\right\} \\
& =\Sigma^{G W}+\left[\left\{\Sigma^{G W}+\operatorname{PCM}\left(\epsilon_{\infty}\right)\right\}-\Sigma^{G W}\right] \\
& \simeq \Sigma^{G W}+\left[\left\{\Sigma^{\operatorname{COHSEX}}+\operatorname{PCM}\left(\epsilon_{\infty}\right)\right\}-\Sigma^{\mathrm{COHSEX}}\right] .
\end{aligned}
$$

This formula approximates the $\{G W+\mathrm{PCM}\}$ calculation by a "gas phase" $G W$ calculation (see below for more details), plus a PCM correction that is calculated at the $\Delta \Sigma^{\mathrm{COHSEX}}$ level. The reason for such a formulation is that the use of the COHSEX approximation in the form of a difference allows to reduce the error introduced by replacing the frequencydependent optical dielectric constant by its low-frequency limit. Even though the screening potential $W$ in the quantum mechanical region is modified by the PCM response, one here assumes that the "dynamical screening error" entering in the difference $\left(\left\{\Sigma^{G W}+\operatorname{PCM}\left(\epsilon_{\infty}\right)\right\}-\left\{\Sigma^{\mathrm{COHSEX}}+\operatorname{PCM}\left(\epsilon_{\infty}\right)\right\}\right)$ largely cancels with the error in $\left(\Sigma^{G W}-\Sigma^{\mathrm{COHSEX}}\right)$.

The adiabatic or "static screening" COHSEX representation of the $G W$ self-energy was originally introduced from the time representation of $G, W$, and $\Sigma .{ }^{18,22,76}$ Using the spectral representations of $G$ and $W,{ }^{20}$ one can readily obtain the following expression in the limit of static screening only:

$$
\begin{aligned}
\Sigma_{\text {static }}^{\text {SEX }}\left(\mathbf{r}, \mathbf{r}^{\prime}\right) & =-\sum_{n}^{o c c} \phi_{n}(\mathbf{r}) \phi_{n}^{*}\left(\mathbf{r}^{\prime}\right) W\left(\mathbf{r}, \mathbf{r}^{\prime} ; \omega=0\right), \\
\Sigma_{\text {static }}^{\mathrm{COH}}\left(\mathbf{r}, \mathbf{r}^{\prime}\right) & =\frac{1}{2} \sum_{n} \phi_{n}(\mathbf{r}) \phi_{n}^{*}\left(\mathbf{r}^{\prime}\right)(W-v)\left(\mathbf{r}, \mathbf{r}^{\prime} ; \omega=0\right) \\
& =\frac{1}{2} \delta\left(\mathbf{r}-\mathbf{r}^{\prime}\right)(W-v)\left(\mathbf{r}, \mathbf{r}^{\prime} ; \omega=0\right) .
\end{aligned}
$$

The contribution $\Sigma^{\mathrm{SEX}}$, which involves a summation over the occupied states only, originates from the poles of $G$. It is called the screened exchange interaction in analogy to the bare exchange term that can be obtained by replacing $W$ with the energy-independent bare Coulomb potential $v$. $\Sigma^{\mathrm{COH}}$ originates from the poles of $W$ and represents the Coulombhole contribution, since it can be shown to be related to the interaction of an electron with its related adiabatically built correlation hole.

\section{A "non-equilibrium" embedded GW calculation in practice}

An important aspect in the use of any polarizable model is the distinction that must be made between screening properties in the ground state and the screening of "fast" electronic excitations. For example, let us consider a molecule with a permanent non-zero dipole or quadrupole in its ground-state. Considering water as solvent, water molecules will respond to the solute ground-state multipoles by a specific average rearrangement of the water molecules in the surrounding of the solute. This involves both the "slow" (solvent nuclear motions) and the "fast" (solvent electronic motions) degrees of freedom that correspond to a total dielectric constant, $\epsilon$, of about 78.35. In contrast, during a vertical electronic excitation out of the ground-state in photoemission or optical experiments, only the "fast" solvent degrees of freedom can react to the solute excitation and one must then consider the $\epsilon_{\infty} \sim 1.78$ solute optical dielectric constant. A non-equilibrium scheme labels a model adapted to treat phenomena too rapid to allow solvent nuclear relaxation, and therefore requires an approach that can discriminate between these different screening mechanisms. On the contrary, the equilibrium limit considers the same dielectric constant for the two processes. Clearly, the non-equilibrium limit corresponds to the correct physical situation when comparing theoretical and experimental vertical excitation energies.

The use of input DFT Kohn-Sham eigenstates as starting point for $G W$ calculations allows a straightforward implementation of non-equilibrium effects. Indeed, the starting DFT calculation can be performed with the PCM based on the static dielectric constant $(\epsilon=78.35$ in water), generating Kohn-Sham eigenstates that account for the 
response of both the slow and fast degrees of freedom. Such a step is now standard in many quantum chemistry codes such as the Gaussian ${ }^{69}$ and $\mathrm{NWchem}^{77}$ codes we use, even though differences in PCM implementations exist (see below). Once the Kohn-Sham eigenstates, that contain the effect of PCM polarization in the ground-state, are generated, one can proceed with the $G W$ calculations that we combine with the PCM approach using the optical dielectric constant $\left(\epsilon_{\infty} \sim 1.78\right)$ in the construction of the screened Coulomb potential $W$ (namely in the construction of $\tilde{v}$ as explained above). As such, vertical excitation energies will only be screened by the dielectric properties of the solvent in the optical range.

In practice, labelling $\left\{\phi_{n}^{\mathrm{PCM}, \epsilon}\right\}$ the Kohn-Sham eigenstates generated by a DFT calculation combined with a $\operatorname{PCM}(\epsilon=78.35)$ polarizable medium, the non-equilibrium solvatochromic shift $P E_{n}$ of the (vertical) quasiparticle energies, namely the solvent induced shift of the occupied or virtual energy levels, read for the $\Delta G W$ approach,

$$
P E_{n}=E_{n}^{G W+\mathrm{PCM}\left(\epsilon_{\infty}\right)}\left[\phi_{n}^{\mathrm{PCM}, \epsilon}\right]-E_{n}^{G W}\left[\phi_{n}^{\mathrm{vac}}\right],
$$

where $E_{n}^{G W+\mathrm{PCM}\left(\epsilon_{\infty}\right)}\left[\phi_{n}^{\mathrm{PCM}, \epsilon}\right]$ are the quasiparticle energies given by a $G W$ calculation combined with the $\operatorname{PCM}\left(\epsilon_{\infty}\right)$ and starting from $\left\{\phi_{n}^{\mathrm{PCM}, \epsilon}\right\}$ Kohn-Sham eigenstates generated with a $\operatorname{PCM}(\epsilon)$ embedding. The $E_{n}^{G W}\left[\phi_{n}^{\text {vac }}\right]$ are gas phase $G W$ quasiparticle energies. Concerning now the $\triangle \mathrm{COHSEX}$ scheme, one obtains

$$
\begin{aligned}
P E_{n} & =E_{n}^{G W}\left[\phi_{n}^{\mathrm{PCM}, \epsilon}\right]-E_{n}^{G W}\left[\phi_{n}^{\mathrm{vac}}\right]+\Delta \Sigma_{\epsilon_{\infty}}^{\mathrm{COHSEX}}, \\
\Delta \Sigma_{\epsilon_{\infty}}^{\mathrm{COHSEX}} & =\left\langle\phi_{n}^{\mathrm{PCM}, \epsilon}\left|\Sigma_{\mathrm{PCM} \epsilon_{\infty}}^{\mathrm{COHSEX}}-\Sigma_{\mathrm{vac}}^{\mathrm{COHSEX}}\right| \phi_{n}^{\mathrm{PCM}, \epsilon}\right\rangle,
\end{aligned}
$$

where the $E_{n}^{G W}\left[\phi_{n}^{\mathrm{PCM}, \epsilon}\right]$ are gas phase $G W$ quasiparticle energies starting from DFT Kohn-Sham eigenstates generated with

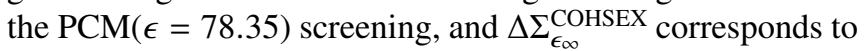
the shift induced at the COHSEX level by the screening of the electronic excitation within the $\operatorname{PCM}\left(\epsilon_{\infty}=1.78\right)$ approach. In Eq. (25), the COHSEX operators are built with the $\left\{\varepsilon_{n}^{\mathrm{PCM}, \epsilon}, \phi_{n}^{\mathrm{PCM}, \epsilon}\right\}$ spectrum containing the information from the screening in the ground-state.

To conclude, we notice that our polarization energies $P E_{n}$ are state-specific since they clearly differ from one level to another as indexed by (n). Further, as emphasized here above, contrary to $\triangle \mathrm{SCF}$ calculations where only frontier orbital polarization shifts can be easily obtained, the present scheme allows to directly determine the shifts of all energy levels. However, as seen in the above formulas, the use of the "frozen" $\left\{\psi_{n}^{\mathrm{PCM}, \epsilon}\right\}$ Kohn-Sham orbitals allows to include ground-state orbital-relaxation effects, but neglects the orbital relaxation induced by the excitation process.

\section{APPLICATION TO NUCLEOBASES}

To validate the present implementation, we study the case of hydrated nucleobases. The importance of understanding the properties of DNA nucleobases in water, namely in an environment close to physiological conditions compared to the dilute gas phase limit, has indeed generated many studies combining standard quantum mechanical approaches with both implicit or explicit descriptions of the aqueous medium.
We refer the reader to a recent review on the subject. ${ }^{78}$ In particular, recent theoretical studies combining $\triangle \mathrm{SCF}$ approaches with the PCM, at the $\mathrm{DFT}^{79}$ or MP2 ${ }^{80}$ levels, may serve as guidelines to validate the present implementation. Further, we perform additional $\triangle \mathrm{SCF}$ calculations at the CCSD level combined with the PCM as another set of reference data. As emphasized in Ref. 80 where experimental values for the vertical ionization potential of hydrated nucleosides were obtained by UV photoelectron spectroscopy, the direct comparison of calculated solvation energies within the PCM approach may be off by a few tenths of an $\mathrm{eV}$, an error that can be reduced by adding a few layers of explicit water molecules. The present study does not therefore aim at obtaining perfect agreement with the (scarce) experimental data, but rather to validate the combination of the $G W$ formalism with the PCM, in comparison with well-established DFT or CCSD techniques. In that respect, the case of water, with a dramatic change in dielectric constant between the slow (nuclear) to the fast (electronic) time scales, offers a stringent test for discriminating between equilibrium and non-equilibrium limits.

\section{A. Computational details}

Our $G W$ calculations are performed with the FIESTA code $^{30,31,58}$ that implements the $G W$ formalism within a Gaussian basis and resolution-of-the-identity approach. The energy integration required to calculate the $\Sigma^{G W}$ self-energy operator (Eq. (3)) is performed along the imaginary axis thanks to a contour deformation approach that does not involve any plasmon-pole approximation nor analytic continuation to the real-axis. The implemented "Coulomb-metric" RI-V replaces four-center two-electrons Coulomb integrals by a sum over three-centers two-electrons integrals, involving the use of an auxiliary basis, labeled $\{\beta\}$. The input KohnSham eigenstates needed to build $G$ and $W$ are generated with the cc-pVTZ correlation-consistent atomic basis set of Dunning. ${ }^{81}$ The corresponding cc-pVTZ-RI auxiliary basis defined by Weigend and co-workers ${ }^{82}$ is adopted for our RI implementation of the $G W$ formalism. As shown in the supplementary material, ${ }^{83}$ the ionization potential solventinduced shift is well converged with the cc-pVTZ basis set, both at the DFT and CCSD levels. Consequently, we adopt this atomic basis for the $G W$ calculations. The adenine, cytosine, thymine, and uracil geometries are the gas-phase MP2/6-31G(d) geometries supplied in Ref. 84.

The reference $\triangle \mathrm{SCF}$ calculations, within DFT or CCSD, are performed with the Gaussian09 package $^{69}$ combined with the IEF-PCM approach. For the DFT and CCSD calculations, the equilibrium ionization potentials (IPs) were obtained by considering independently the neutral and cationic forms in a fully relaxed solvent. In the non-equilibrium approach, the response to the dipole, quadrupole, etc. of the neutral molecule in its ground-state is calculated with the $\operatorname{PCM}(\epsilon \simeq 78.35)$ model, generating a first set of "static" surface charges. The polarisation energy associated with the changes in charge density from the neutral molecule to its cation is then treated with the $\operatorname{PCM}\left(\epsilon_{\infty}=1.78\right)$ medium, generating additional "dynamical" charges. These two sets of induced charges (static 
and dynamical) contribute to the non-equilibrium solvation energy. ${ }^{85}$

The input Kohn-Sham eigenstates needed to start the $G W$ calculations are generated by the NWChem package. ${ }^{77}$ NWChem does not allow the use of the IEF-PCM formulation but implements instead the COSMO. While much discussions can be found on the differences between the IEF-PCM and COSMO implementations in the limit in particular of apolar solvents with small dielectric constant ${ }^{86}$ the case of water, a solvent with a very large dielectric response, is an ideal scenario where the two continuum implementations should provide extremely close results. We emphasize here that the (NWChem+COSMO) calculations are only needed for the ground-state involving the very large $(\epsilon \simeq 78)$ dielectric constant, while the screening of the electronic excitation with the optical dielectric constant $\left(\epsilon_{\infty} \simeq 1.78\right)$ is performed with the FIESTA PCM implementation that solves exactly Poisson's equation inside and outside the cavity. As a simple test, we compared, within the same code (Gaussian09), the $\triangle$ SCF PBE0/cc-pVTZ ionization potential of adenine in water determined with the IEF-PCM or C-PCM implementations, with values of $6.26 \mathrm{eV}$ in both implementations for equilibrium calculations, and values of $7.41 \mathrm{eV}$ and $7.40 \mathrm{eV}$ in the nonequilibrium calculations, with the C-PCM and IEF-PCM, respectively. Such differences of the order of $10 \mathrm{meV}$ are marginal as compared to the absolute solvatochromic shift that attains $0.74 \mathrm{eV}$ (the IP is $8.15 \mathrm{eV}$ in gas phase). Even though the C-PCM implementation in Gaussian09 ${ }^{87}$ and the COSMO implementation in NWChem may not rely on strictly equivalent parameters for setting up the cavity, they both implement essentially the same (scaled) conductor condition boundaries. ${ }^{11,87}$

A description of the PCM implementation in the Fiesta code is provided in the Appendix and in the supplementary material ${ }^{83}$ It closely follows the original IEFPCM formulation, ${ }^{5,6}$ modified in order to account exactly for source $\{\beta\}$ and test $\left\{\beta^{\prime}\right\}$ auxiliary basis orbitals spilling out of the cavity. For the sake of illustration, we plot here in Fig. 3 a representation of the cavity around the adenine molecule, together with an isocontour description of the HOMO KohnSham orbital. The molecular cavity is built from the standard superposition of atom-centered spheres with (scaled) universal

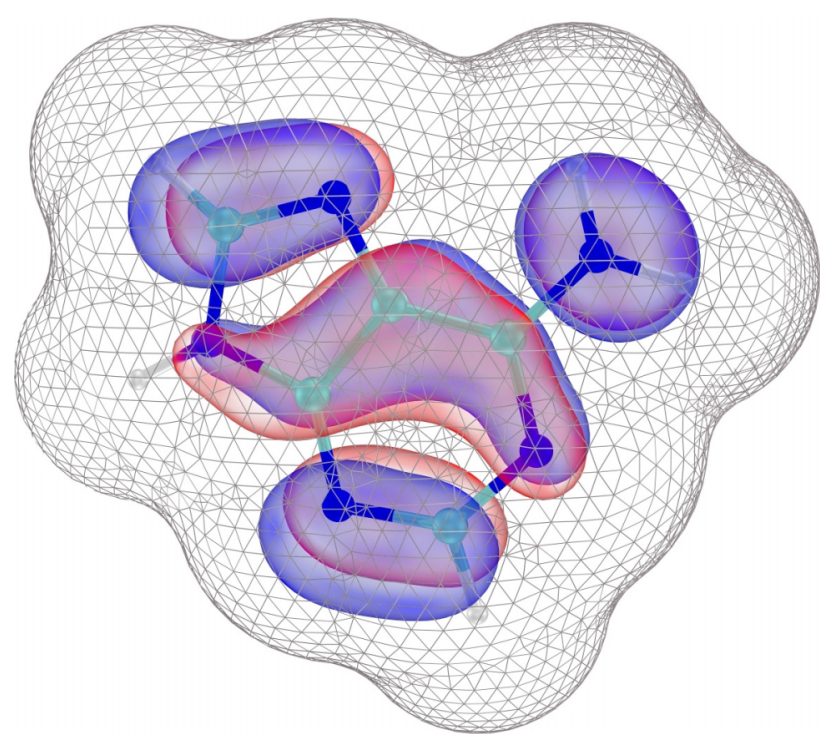

FIG. 3. Representation of the molecular cavity generated for the adenine molecule using van der Waal's radii with a 1.1 scaling factor. The discretization of the (smoothed) surface is also shown, corresponding to an initial tesselation of 1280 tesserae per atomic sphere. The final surface contains 6756 tesserae. For sake of illustration, an isocontour representation of the HOMO Kohn-Sham (PBE0) molecular orbital is shown. More details about the calculation of the reaction field is provided in the Appendix and in the supplementary material. ${ }^{83}$

force field $^{88}$ van der Waal's radii, adopting a 1.1 scaling factor consistently with the default in Gaussian09. The obtained surface is smoothed at the atomic sphere intersections in order to avoid too large local curvature. The convergency with respect to the discretisation of the surface was carefully checked. In the case of a spherical cavity, the renormalization by the reaction field of the Coulomb integrals between auxiliary basis orbitals was shown to match very precisely exact analytic values (see supplementary material). ${ }^{83}$

\section{B. Results}

Before addressing the effect of the solvent reaction field, we compare in Table I the $G W$ formalism with several $\triangle \mathrm{SCF}$ schemes for the IP of adenine, cytosine, thymine, and uracil in

TABLE I. Calculated gas phase vertical ionization potential. $\triangle \mathrm{SCF}$ and $G W$ calculations performed at the $c c$-pVTZ level. The PMP2 data are the projected MP2/aug-cc-pVDZ data as calculated by Slavícek et al. in Ref. 80. The abbreviation CAM stands for the CAM-B3LYP functional. The last column contains the range of experimental values from Refs. 90-93. All values are in eV.

\begin{tabular}{|c|c|c|c|c|c|c|c|c|c|}
\hline & \multirow[b]{2}{*}{$\mathrm{PMP}^{\mathrm{a}}$} & \multirow[b]{2}{*}{$\triangle \mathrm{PBE} 0$} & \multirow[b]{2}{*}{$\triangle \mathrm{CAM}$} & \multirow[b]{2}{*}{$\Delta \mathrm{CCSD}$} & \multicolumn{2}{|c|}{$G_{0} W_{0}$} & \multicolumn{2}{|c|}{$\mathrm{ev} G W$} & \multirow[b]{2}{*}{ Expt. ${ }^{b, c, d, e}$} \\
\hline & & & & & PBE0 & CAM & PBE0 & CAM & \\
\hline Adenine & & 8.15 & 8.26 & 8.24 & 7.99 & 8.21 & 8.21 & 8.28 & $8.3-8.5$ \\
\hline Cytosine & 8.84 & 8.60 & 8.74 & 8.68 & 8.44 & 8.71 & 8.77 & 8.82 & $8.8-9.0$ \\
\hline Thymine & 9.15 & 8.93 & 9.06 & 9.04 & 8.86 & 9.11 & 9.13 & 9.21 & $9.0-9.2$ \\
\hline Uracil & & 9.39 & 9.52 & 9.44 & 9.25 & 9.50 & 9.54 & 9.60 & 9.4-9.6 \\
\hline
\end{tabular}

\footnotetext{
${ }^{a}$ Reference 80.

${ }^{\mathrm{b}}$ Reference 90.

${ }^{\mathrm{c}}$ Compiled in Ref. 91.

${ }^{\mathrm{d}}$ Compiled in Ref. 92.

${ }^{\mathrm{e}}$ Reference 93.
} 
the gas phase. While differences exist between all approaches, it can be seen that the $\operatorname{ev} G W$ formalism, with partial self-consistency on the eigenvalues (see Sec. II) compares very well with the $\triangle \mathrm{PBE} 0, \triangle \mathrm{CAM}-\mathrm{B} 3 \mathrm{LYP}$, and $\triangle \mathrm{CCSD}$ results. The non-self-consistent $G_{0} W_{0}$ calculations starting from PBE0 Kohn-Sham eigenstates tend to underestimate the ionization potential, so that in the following of the paper our $G W$ calculations are systematically performed at the evGW level. Even though both CCSD and $G W$ ionization potential may still increase by $0.1-0.2 \mathrm{eV}$ upon increasing the size of the Kohn-Sham atomic basis set (see the supplementary material), ${ }^{83}$ we see that the agreement between all techniques and the experimentally available data is already very satisfying. ${ }^{89}$ The same quality of agreement was attained in previously published projected MP2/aug-ccpVDZ calculations by Slavícek and co-workers. ${ }^{80}$ Similarly, in the case of cytosine, the present numbers are close to the $8.98 \mathrm{eV}$ value obtained at the $\Delta \mathrm{M} 05-2 \mathrm{X} / 6-31+\mathrm{G}(\mathrm{d}, \mathrm{p})$ level by Muñoz-Losa and co-workers in Ref. 79.

Let us now turn to the solvent-induced shifts that are listed in Table II for the ionization potential calculated at the cc-pVTZ level within the $\triangle$ SCF approaches (DFT and CCSD) and with the present $G W$ formalism. We also include the PMP2/cc-pVDZ data by Slavî́cek et al. ${ }^{80}$ for comparisons. We present both the non-equilibrium shifts, considering the static dielectric constant for the ground-state DFT calculations and its optical counterpart for the $G W$ runs, and the equilibrium values for which the $\epsilon \sim 78.35$ water dielectric constant, including both nuclear and electronic degrees of freedom, has been used throughout the calculation. Our results are also summarized in Figure 4.

Our non-equilibrium PBE0 and CAM-B3LYP ionization potential solvatochromic shifts for cytosine, $-0.76 \mathrm{eV}$ and $-0.78 \mathrm{eV}$, respectively, compare very well with the $-0.78 \mathrm{eV}$ value obtained at the M05-2X level by Muñoz-Losa and co-workers. ${ }^{79}$ Clearly, by comparing the $\triangle \mathrm{SCF}$ data based on PBE0 or CAM-B3LYP exchange-correlation functionals, one

TABLE II. Calculated solvatochromic energy shifts of the ionization potential. We report the absolute value, the shift being always negative. $\triangle \mathrm{SCF}$ and $G W$ calculations performed at the $c c$-pVTZ level. The PMP2 data are the projected MP2 data combined with the PCM as calculated by Slavícek et al. in Ref. 80. All values are in eV.

\begin{tabular}{|c|c|c|c|c|c|c|c|}
\hline & \multirow[b]{2}{*}{$\mathrm{PMP}^{\mathrm{a}}$} & \multirow[b]{2}{*}{ PBE0 } & \multirow[b]{2}{*}{ CAM } & \multirow[b]{2}{*}{ CCSD } & \multirow{2}{*}{$\begin{array}{l}\triangle G W \\
\text { PBE0 }\end{array}$} & \multicolumn{2}{|c|}{$\triangle \mathrm{COHSEX}$} \\
\hline & & & & & & PBE0 & CAM \\
\hline \multicolumn{8}{|c|}{ Non-equilibrium calculations } \\
\hline Adenine & & 0.74 & 0.76 & 0.74 & 0.60 & 0.64 & 0.65 \\
\hline Cytosine & 0.98 & 0.76 & 0.78 & 0.78 & 0.67 & 0.70 & 0.70 \\
\hline Thymine & 1.28 & 1.04 & 1.07 & 1.10 & 0.95 & 0.99 & 1.01 \\
\hline Uracil & & 1.12 & 1.15 & 1.18 & 1.05 & 1.07 & 1.10 \\
\hline \multicolumn{8}{|c|}{ Equilibrium calculations } \\
\hline Adenine & & 1.89 & 1.90 & 1.90 & 1.51 & 1.64 & 1.65 \\
\hline Cytosine & 2.25 & 1.98 & 2.00 & 2.00 & 1.67 & 1.75 & 1.75 \\
\hline Thymine & 2.50 & 2.22 & 2.26 & 2.26 & 1.92 & 2.03 & 2.05 \\
\hline Uracil & & 2.36 & 2.40 & 2.40 & 1.96 & 2.15 & 2.17 \\
\hline
\end{tabular}

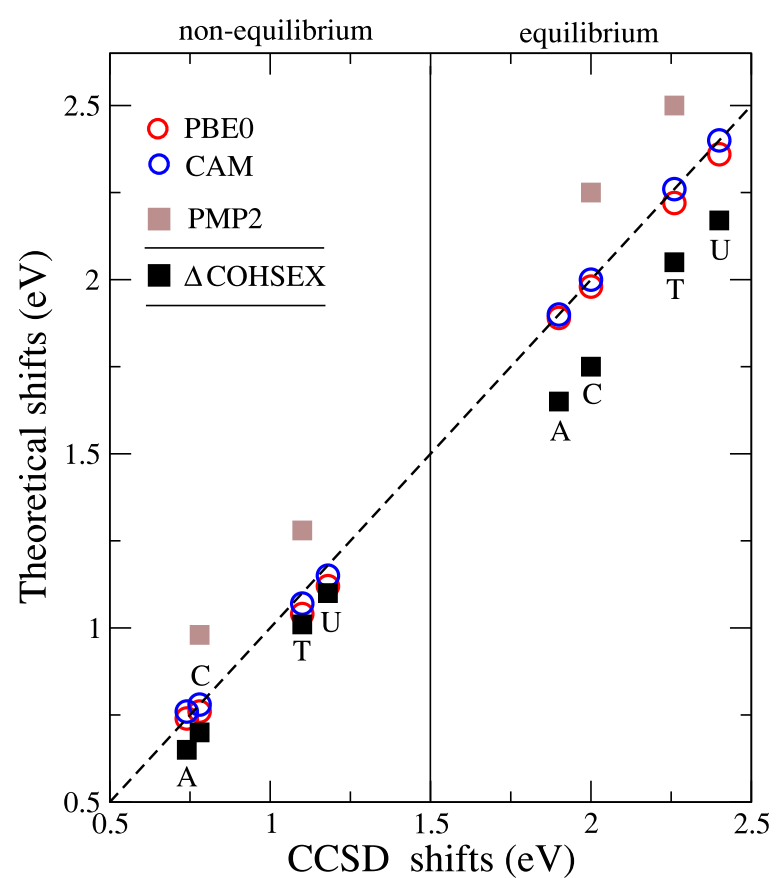

FIG. 4. Theoretical solvent (water) induced shifts of the HOMO energy for nucleobases within the non-equilibrium and equilibrium solvation limits. The reference energy shifts are taken to be the (CCSD+IEF-PCM) data indicated by the dashed first diagonal. The PMP2 values are the projected MP2/aug-cc-pVDZ data as calculated by Slavícek et al. in Ref. 80. The letters A, C, T and U, stand for adenine, cytosine, thymine and uracil, respectively.

notices 20-30 meV variations only. These differences are small compared to the absolute shift value. Similar conclusions can be obtained when comparing CCSD data with DFT ones. The CCSD shifts are, on the average, slightly larger than the PBE0 one, but again with maximum differences of the order of $60 \mathrm{meV}$ in the case of thymine and uracil, that represents a $\sim 6 \%$ maximum discrepancy. By comparison, the PMP2 values of Ref. 80 stand systematically above both the DFT and CCSD $\triangle \mathrm{SCF}$ shifts, with differences of the order of $0.2 \mathrm{eV}$, that correspond to ca. $20 \%$ deviations. As expected, the equilibrium limit yields much larger effects than the non-equilibrium one. This is obviously related to the use of the very large $\epsilon \sim 78.35$ solvent dielectric constant that strongly over-stabilizes the cationic species. The differences between PMP2 and CCSD data slightly increase to about $0.25 \mathrm{eV}$ in absolute value, leading interestingly to a smaller $\sim 7.5 \%$ percentage of error.

We now turn to the solvatochromic shifts obtained at the $G W$ level. The overall tendency is that both the $\Delta G W$ and $\triangle$ COHSEX values are smaller than their DFT counterparts. In the case of non-equilibrium calculations, the scheme of choice when discussing vertical energies, the $\triangle$ COHSEX@PBE0 approach (black squares in Fig. 4) yields shifts that are on the average $65 \mathrm{meV}$ smaller than the $\triangle \mathrm{PBE} 0$ values, corresponding to an average difference of $7 \%$. The situation is nearly identical when comparing $\triangle$ COHSEX@CAM-B3LYP to $\triangle$ CAM-B3LYP calculations, with a $70 \mathrm{meV}$ average discrepancy. These variations are comparable to the one obtained when comparing CCSD and PBE0 $\triangle \mathrm{SCF}$ values. The straightforward application of the $\Delta \mathrm{GW}$ scheme leads to a larger $\sim 0.1 \mathrm{eV}$ discrepancy, comparing the $\triangle \mathrm{GW} @ \mathrm{PBE} 0$ 
with the corresponding DFT $\triangle \mathrm{PBE} 0$ values, corresponding to a larger $\sim 11 \%$ difference in the energy shift. Such deviations can be compared to the PMP2 data ${ }^{80}$ that yield larger discrepancies (see brown squares in Fig. 4, non-equilibrium limit).

The same trends can be observed in the case of the equilibrium calculations. Clearly, the difference compared to the reference $\triangle C C S D$ values is larger in absolute value (see Fig. 4). The comparison between $\triangle$ COHSEX@PBE0 and $\triangle \mathrm{PBE} 0$ data leads to an average difference of $0.22 \mathrm{eV}$ for an average shift of $2.11 \mathrm{eV}$, that is, a $\sim 10 \%$ difference, to be compared to the $\sim 7 \%$ relative deviation for the non-equilibrium calculations. Concerning the $\Delta G W$ scheme, the shifts are again seen to be smaller, bringing the average difference between $\triangle G W @$ PBE0 and $\triangle \mathrm{PBE} 0$ to $0.34 \mathrm{eV}$, namely a $16 \%$ deviation. This can be viewed as a sizable deviation, larger than the $11 \%$ discrepancy of PMP2 compared to CCSD, indicating, as justified here above, that the $\triangle$ COHSEX scheme is a favorable approach for the combination of the $G W$ formalism with the present frequencyindependent PCM response.

\section{DISCUSSION}

We start the present discussion by emphasizing again the differences between the approaches compared in this work. In the $\triangle \mathrm{SCF}$ techniques chosen as references, the energy shift is obtained as a difference between two self-consistent groundstate total energy calculations for the $(N)$ and $(N-1)$-electron systems. As such, orbital and charge density relaxation upon addition of a charge and upon screening by the solvent are adequately considered. Further, since the present DFT calculations rely on an adiabatic kernel, the use of a frequencyindependent solvent dielectric response in the optical range does not come as a methodological problem.

The present implementation, aiming at combining the many-body Green's function $G W$ formalism with the standard PCM approach, relies on two main approximations: (a) the neglect of the frequency dependence of the PCM dielectric response in the optical range, and (b) the neglect of the orbital relaxation upon electronic excitations, accounting only for the wavefunction relaxation in the ground-state. More precisely, while the self-energy operator fully accounts for the interaction of the added charge with the $N$-electron system through the calculated polarization response system function, including both the $\mathrm{QM}$ and $\mathrm{PCM}$ response, the added charge is assumed to take the shape of the guest Kohn-Sham orbital as calculated for the $N$-electron system. These approximations can certainly be circumvented but at the cost of both losing the advantage of benefiting from the well-known existing PCM implementations and a very large increase of the computational cost in the case of selfconsistent $G W$ schemes allowing to update the wavefunctions in the ionized state. While practical solutions for such refinements may be explored in the future, it remains that the comparisons performed here with the $\triangle \mathrm{SCF}$ techniques lead to an agreement that does not call for implementing more sophisticated/expensive techniques, at least in this initial stage. Indeed, taking the case of the calculations performed in the physically adequate non-equilibrium limit, the obtained difference between the $\triangle$ COHSEX and the $\triangle \mathrm{SCF}$ values, limited to $\sim 60-70 \mathrm{meV}$, can already be considered as very satisfactory.

To illustrate the difficulty in predicting the effect of generalizing the PCM model to a frequency-dependent optical dielectric constant, we write for illustration the exact ${ }^{20}$ dynamical screened-exchange contribution to the HOMO selfenergy,

$$
\begin{aligned}
& \left\langle\phi_{\mathrm{H}}\left|\Sigma^{\operatorname{SEX}}\left(\varepsilon_{\mathrm{H}}\right)\right| \phi_{\mathrm{H}}\right\rangle \\
& \quad=-\sum_{n}^{o c c}\left\langle\phi_{\mathrm{H}}\left|\phi_{n}(\mathbf{r}) \phi_{n}^{*}\left(\mathbf{r}^{\prime}\right) W\left(\mathbf{r}, \mathbf{r}^{\prime} ; \varepsilon_{\mathrm{H}}-\varepsilon_{n}\right)\right| \phi_{\mathrm{H}}\right\rangle,
\end{aligned}
$$

where $\left\{\varepsilon_{\mathrm{H}}, \phi_{\mathrm{H}}\right\}$ are the HOMO eigenvalue and eigenvector. For molecules such as the DNA nucleobases, the energy difference $\left(\varepsilon_{\mathrm{H}}-\varepsilon_{n}\right)$, where $(n)$ indexes the occupied valence orbitals, spans typically $20 \mathrm{eV}$. Over this energy range, the macroscopic optical dielectric constant of water takes values that are both larger and smaller than the low-frequency $\epsilon_{\infty} \sim 1.78$ limit (see Fig. 2). As such, it cannot be concluded straightforwardly whether the restriction to the low-frequency limit results in underestimating or overestimating the effect of the reaction field.

It is however interesting to compare the $\Delta G W$ and the $G W+\Delta C O H S E X$ schemes. The discrepancy between the two formulations goes from an average of $35 \mathrm{meV}$ in the nonequilibrium case to $100 \mathrm{meV}$ in the equilibrium limit. Such variations amount respectively to $4.1 \%$ and $5.3 \%$ as compared to the average $\triangle$ COHSEX@PBE0 energy shift. As such, the differences remain rather small but of the same order of magnitude as the difference between the $G W+\Delta \mathrm{COHSEX}$ and the $\triangle \mathrm{SCF}$ results. This hints that the neglect of the dynamical nature of the screening properties of the solute in the optical range, and the way it is handled, may contribute significantly to the observed differences.

We now explore the issue of orbital relaxation, recalling that in the present $G W$ formalism, the Kohn-Sham wavefunctions are frozen and not updated self-consistently. While we cannot explore the effect of orbital self-consistent relaxation within $G W$, we explore the case of the "default" $(\mathrm{PCM}+\Delta \mathrm{SCF})$ scheme combined with CCSD formalism, for which the PCM responds to the Hartree-Fock charge density of the neutral and cation molecules. Namely, the PCM response is not consistent with the charge-density corresponding to the CCSD many-body wave function ansatz. This corresponds to the so-called unrelaxed situation. For uracil (equilibrium limit), we have evaluated the impact of determining selfconsistently the solvent effects for the CCSD density (socalled externaliteration procedure in Gaussian09) and found only small variations, as the IP goes from $7.03 \mathrm{eV}$ to $7.04 \mathrm{eV}$. As such, orbital relaxation, even in the equilibrium limit where the impact of solvation is over-emphasized, does not contribute by more than $10 \mathrm{meV}$ to the energy shift.

Finally, we also test the influence of the size of the cavity by building a smaller one, adopting atomic spheres of radius equal to the standard van der Waals radii, dropping the 1.1 rescaling factor. In the case of starting CAMB3LYP Kohn-Sham eigenstates, we obtain non-equilibrium $(G W+\Delta$ COHSEX) solvent-induced shifts of $0.69 \mathrm{eV}, 0.72 \mathrm{eV}$, 
$1.02 \mathrm{eV}$, and $1.12 \mathrm{eV}$ for adenine, cytosine, thymine, and uracil, respectively, which are on the average only $\sim 22 \mathrm{meV}$ larger than that reported in the last column of Table II for the standard cavity size. Such variations associated with a significant cavity volume change are not negligible, but are not so large that they can account for the differences between the $\triangle \mathrm{SCF}$ and $(G W+\Delta \mathrm{COHSEX})$ schemes. As such, the possible differences in the construction of the cavity are not expected to contribute much to the differences observed between the different approaches.

To close this discussion, we emphasize again that the present implementation allows to calculate the solventinduced shift related to all electronic addition or removal energies, namely the specific polarization energies associated with transitions from the $N$-electron ground-state to any eigenstate of the $(N+1)$ and $(N-1)$ electron systems. For the sake of illustration, we plot in Fig. 5 the (non-equilibrium) solvatochromic energy shift associated with a large set of occupied and virtual energy levels for the uracil molecule. Clearly, there is a large spread in the $(G W+\Delta$ COHSEX) polarization energies (blue circles). However, at least for the occupied states (quasiparticle energies below $-5 \mathrm{eV}$ ), most of the spread comes from the effect of the solvent at the ground-state level, namely the effect of the PCM $(\epsilon \simeq 78)$ on the input Kohn-Sham eigenvalues (black crosses). Such an observation holds much less in the case of virtual (unoccupied) levels probably due to the increase in delocalization for empty states with large positive energy. The shift of the KohnSham DFT energy levels is related to the polarization of the PCM environment in response to the field generated by the nucleobases ground-state dipole. The electrostatic potential

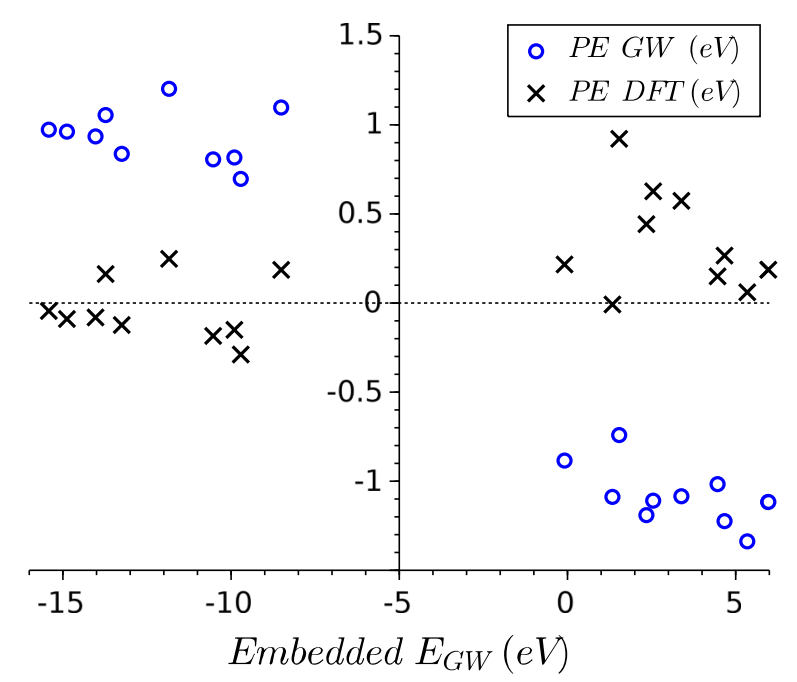

FIG. 5. Solvent-induced non-equilibrium polarization energies (PE) for 10 occupied and 10 virtual energy levels of the uracil molecule. The (blue) circles are the $(G W+\Delta$ COHSEX) polarization energies plotted with respect to the absolute $(G W+\Delta C O H S E X)$ energies in solution. The black crosses indicate the solvent-induced shift at the (DFT+PCM) (PBE0) level for the neutral molecule. The Kohn-Sham (DFT+PCM) calculations performed on the neutral molecule cannot give the overall solvent-induced shift but contribute significantly to the spread in polarization energy amongst, e.g., the occupied states. Notice that the shift of the occupied/virtual $G W$ energy levels is of opposite signs as expected, leading to a $\sim 2 \mathrm{eV}$ reduction of the HOMO-LUMO gap upon solvation. generated by the PCM polarization charges acts differently on Kohn-Sham orbitals with different spatial shape. Since the reaction field generated by the ground-state dipole is much smaller than the one related to adding a charge on the QM system, the energy shifts calculated at the Kohn-Sham DFT level are much smaller than the one calculated with the $G W$ or $\triangle \mathrm{SCF}$ formalisms.

\section{CONCLUSIONS AND OUTLOOK}

We have implemented the polarizable continuum model in the framework of the many-body Green's function $G W$ formalism for the calculation of the vertical electronic addition and removal energies in solution. We have demonstrated in particular that the solvatochromic shifts agree quantitatively with the standard combination of the PCM formalism with $\triangle \mathrm{SCF}$ techniques at the DFT and CCSD levels, with differences of the order of a few percents only, corresponding to deviations of the order of $\sim 70 \mathrm{meV}$ out of a shift of about $1 \mathrm{eV}$ for non-equilibrium PCM calculations. Such small variations are expected to come from the approximation that consists of merging the $G W$ formalism, which accounts for the frequency-dependence of the dielectric response in the optical range, with standard PCM models that only consider the low-frequency optical dielectric response to electronic excitations. Such conclusions were validated in the case of the evolution of the ionization potential of nucleobases from the gas to the aqueous phase. The possibility of determining solvent effects in both the equilibrium and non-equilibrium limits was clearly illustrated. Further, beyond the case of frontier orbitals, the energy shift of all occupied/virtual energy levels can be obtained on the same footing. The extension of the present implementation to the Bethe-Salpeter equation formalism for the calculation of optical excitations in solvated condition is under way, together with the generalization of the present scheme to the combination of the $G W$ formalism with discrete polarizable models, ${ }^{94}$ for the calculation of the polarization energies in organic crystals with applications to organic light-emitting and photovoltaic devices.

\section{ACKNOWLEDGMENTS}

The authors are indebted to Eric Cancès and B. Mennucci for discussions and guidance concerning the IEF-PCM formalism and the implementation of non-equilibrium DFT $\triangle \mathrm{SCF}$ calculations in the Gaussian package. I.D. and X.B. acknowledge Gabriele D'Avino for strategical discussions concerning future extensions to discrete polarizable models. This project has received funding from the European Union Horizon 2020 research and innovation programme under Grant agreement No 646176 (EXTMOS) and No 676629 (EoCoE). X.B. acknowledges funding from the French No. ANR-12BS04-0001 "PANELS." D.J. acknowledges the European Research Council (ERC) and the Région des Pays de la Loire for financial support in the framework of a Starting Grant (No. Marches-278845) and the LumoMat RFI project, respectively. This research used resources of: (i) the GENCICINES/IDRIS; (ii) CCIPL (Centre de Calcul Intensif des Pays 
de Loire); (iii) a local Troy cluster and (iv) HPC resources from ArronaxPlus (Grant No. ANR-11-EQPX-0004 funded by the French National Agency for Research).

\section{APPENDIX: CAVITY REACTION FIELD}

Following the original IEF-PCM formulation notations, 5,6 we consider a cavity defined by the closed surface $\Gamma$ delimiting two open domains $\Omega_{i}$ and $\Omega_{e}$ (respectively, internal and external), and a source charge $\rho(\mathbf{r})$. We thus look for the potential $V(\mathbf{r})$ solution of the following Poisson's equation:

$$
\nabla\{\epsilon(\mathbf{r}) \nabla V(\mathbf{r})\}=\rho(\mathbf{r}), \quad \epsilon(\mathbf{r})=\left\{\begin{array}{l}
\epsilon_{i} \text { when } \mathbf{r} \in \Omega_{i} \\
\epsilon_{e} \text { when } \mathbf{r} \in \Omega_{e}
\end{array} .\right.
$$

We will first consider the case of a source charge $\rho(\mathbf{r})$ entirely contained inside the $\Omega_{i}$ domain, recovering the original IEFPCM formulation, before considering the modifications to be adopted to treat exactly the case of a source charge spilling out in the $\Omega_{e}$ domain.

\section{Standard IEF-PCM formulation}

Following Cancès and co-workers, 5,6 we consider first the case of a source charge $\rho$ entirely contained within the $\Omega_{i}$ domain. In this case, a possible functional form for the potential $V(\mathbf{r})$ solution of Poisson's equation in both $\Omega_{i}$ and $\Omega_{e}$ is

$$
\begin{aligned}
\forall \mathbf{r} & \in \mathbb{R}^{3} \backslash \Gamma, V(\mathbf{r}) \\
& :=\frac{1}{\epsilon_{i}}\left(\int_{\Omega_{i}} d \mathbf{r}^{\prime} \frac{1}{\left|\mathbf{r}-\mathbf{r}^{\prime}\right|} \rho\left(\mathbf{r}^{\prime}\right)+\int_{\Gamma} d \mathbf{y} \frac{1}{|\mathbf{r}-\mathbf{y}|} \sigma(\mathbf{y})\right) .
\end{aligned}
$$

The surface charge $\sigma$ is determined by the continuity conditions of $V(\mathbf{r})$ across $\Gamma$. Using the following notations:

$$
V_{i / e}(\mathbf{x}):=\lim _{\mathbf{r} \in \Omega_{i / e} \rightarrow \mathbf{x}} V(\mathbf{r})
$$

and denoting $\mathbf{n}(\mathbf{x})$ the normal to $\Gamma$ at point $\mathbf{x} \in \Gamma$, the continuity conditions write

$[V]: \quad \forall \mathbf{x} \in \Gamma, V_{i}(\mathbf{x})=V_{e}(\mathbf{x})$,

$[\partial V]: \quad \forall \mathbf{x} \in \Gamma, \epsilon_{i} \mathbf{n}(\mathbf{x}) \cdot \nabla V_{i}(\mathbf{x})=\epsilon_{e} \mathbf{n}(\mathbf{x}) \cdot \nabla V_{e}(\mathbf{x})$.

Since the surface charge distribution $\sigma$ does not induce any discontinuity of the potential $V(\mathbf{r})$ across $\Gamma$, condition (A2) is automatically fulfilled. Here, we adopt again the notations of Refs. 5 and 6 and write

$$
\left(\mathcal{D}^{*} \cdot \sigma\right)(x)=\int_{\Gamma} d \mathbf{y} \mathbf{n}(\mathbf{x}) \cdot \nabla_{\mathbf{x}} \frac{1}{|\mathbf{x}-\mathbf{y}|} \sigma(\mathbf{y})
$$

the corresponding component of the Calderon projector. ${ }^{95}$ The normal component of the electric field on the $\Omega_{i}$ and $\Omega_{e}$ domain boundaries is given by (see the supplementary material for a sketch of proof $^{83}$ ):

$E_{i / e}(\mathbf{x})=-\mathbf{n}(\mathbf{x}) \cdot \nabla V_{i / e}(\mathbf{x})=-\left(\mathcal{D}^{*} \cdot \sigma\right)(\mathbf{x}) \mp \frac{\sigma(\mathbf{x})}{2}$.

Denoting $E_{n}(\mathbf{x})$ the normal field coming from the initial charge distribution $\rho$ at a point $\mathbf{x} \in \Gamma$, the second continuity condition
(A3) writes

$$
\begin{aligned}
\epsilon_{i}(- & \left.\frac{\sigma(\mathbf{x})}{2}-\left(\mathcal{D}^{*} \cdot \sigma\right)(\mathbf{x})+E_{n}(\mathbf{x})\right) \\
& =\epsilon_{e}\left(\frac{\sigma(\mathbf{x})}{2}-\left(\mathcal{D}^{*} \cdot \sigma\right)(\mathbf{x})+E_{n}(\mathbf{x})\right) .
\end{aligned}
$$

After some simple manipulations, we find again Eq. (2.8) of Ref. 6 defining the surface charge density $\sigma$ as given by the standard IEF-PCM approach,

$$
\left(\frac{\epsilon_{e}+\epsilon_{i}}{\epsilon_{e}-\epsilon_{i}} \frac{\mathbb{I}}{2}-\mathcal{D}^{*}\right) \sigma=-E_{n}
$$

\section{Modified IEF-PCM formulation}

In order to take into account the possibility of having some of the source charge distribution spilling in $\Omega_{e}$, we choose the following formulation of the solution $V(\mathbf{r})$ of the Poisson equation:

$$
V(\mathbf{r}):=\left\{\begin{array}{l}
\frac{1}{\epsilon_{i}} \int_{\mathbb{R}^{3}} d \mathbf{r}^{\prime} \frac{1}{\left|\mathbf{r}-\mathbf{r}^{\prime}\right|} \rho\left(\mathbf{r}^{\prime}\right)+V_{R}(\mathbf{r}), \mathbf{r} \in \Omega_{i} \\
\frac{1}{\epsilon_{e}} \int_{\mathbb{R}^{3}} d \mathbf{r}^{\prime} \frac{1}{\left|\mathbf{r}-\mathbf{r}^{\prime}\right|} \rho\left(\mathbf{r}^{\prime}\right)+V_{R}(\mathbf{r}), \mathbf{r} \in \Omega_{e}
\end{array} .\right.
$$

The source charge distribution $\rho(\mathbf{r})$ is now sampled over $\mathbb{R}^{3}$. The reaction potential $V_{R}(\mathbf{r})$ needs to be defined as the combination of a single and a double-layer potential, namely a charge plus dipole surface distribution,

$V_{R}(\mathbf{r}):=\int_{\Gamma} d \mathbf{y}\left(\frac{1}{|\mathbf{r}-\mathbf{y}|} \sigma(\mathbf{y})+\frac{\mathbf{r}-\mathbf{y}}{|\mathbf{r}-\mathbf{y}|^{3}} \cdot \mathbf{n}(\mathbf{y}) \mu(\mathbf{y})\right)$,

where $\mu(\mathbf{y})$ is the normal component of the surface dipole. Under this form, the potential $V(\mathbf{r})$ satisfies automatically the Poisson equation in both $\Omega_{i}$ and $\Omega_{e}$. The first contribution (single layer potential) to the reaction field $V_{R}(\mathbf{r})$ is identical to the one of the standard IEF-PCM model. The second one (double layer potential) corresponds to the potential issued from a normal surface dipole distribution $\mu$. This dipole distribution is unequivocally related to the discontinuity coming from taking explicitly the dielectric constant into account within the first term of the form (A6). The jump of the reaction potential $V_{R}$ across $\Gamma$ is simply given by

$$
\lim _{\mathbf{r} \in \Omega_{e} \rightarrow \mathbf{x}} V_{R}(\mathbf{r})-\lim _{\mathbf{r} \in \Omega_{i} \rightarrow \mathbf{x}} V_{R}(\mathbf{r})=\mu(\mathbf{x}) .
$$

Keeping the notations previously introduced for $V_{i}$ and $V_{e}$ and introducing $\phi$ the potential issued from the source distribution in vacuum, we have now the following expression for the first continuity condition (A2):

$$
V_{e}(\mathbf{x})-V_{i}(\mathbf{x})=\left(\frac{1}{\epsilon_{e}}-\frac{1}{\epsilon_{i}}\right) \phi(\mathbf{x})+\mu(\mathbf{x})=0
$$

so that one simply has

$$
\mu(\mathbf{x})=\frac{\epsilon_{e}-\epsilon_{i}}{\epsilon_{e} \epsilon_{i}} \phi(\mathbf{x}) .
$$

For the sake of consistency with the IEF-PCM notations, we introduce the following integral operator representation for the electric field (given a minus sign) generated by the surface 
normal dipole distribution $\mu$ at a point $\mathbf{x} \in \Gamma$ :

$$
\left(\mathcal{K}^{*} \cdot \mu\right)(x)=\int_{\Gamma} d \mathbf{y} \mathbf{n}(\mathbf{x}) \cdot \nabla_{\mathbf{x}}\left[\frac{\mathbf{x}-\mathbf{y}}{|\mathbf{x}-\mathbf{y}|^{3}} \cdot \mathbf{n}(\mathbf{y}) \mu(\mathbf{y})\right] .
$$

The second continuity equation (A3) gives then

$$
\left(\frac{\epsilon_{e}+\epsilon_{i}}{\epsilon_{e}-\epsilon_{i}} \frac{\mathbb{I}}{2}-\mathcal{D}^{*}\right) \sigma=-E_{n}+\frac{\epsilon_{e}-\epsilon_{i}}{\epsilon_{e} \epsilon_{i}} \mathcal{K}^{*} \phi .
$$

Again, this representation is unique, since the left member of Eq. (A9) has been shown to lead to a unique solution $\sigma$ in Ref. 6 and $\mu$ is explicitly related to the potential $\phi$. In practice, the tessellation of the surface $\Gamma$ translates Eq. (A9) into a standard linear algebra problem that is solved numerically in order to get $\sigma$, while $\mu$ is directly estimated through $\phi$. We provide in the Supplementary Material ${ }^{83}$ a convergence test of the renormalized Coulomb interaction between two Gaussian charge distributions as a function of the surface tessellation.

To conclude, we note that while the generation of the $V_{R}(\mathbf{r})$ reaction potential generated by a source charge inside and outside the cavity does not represent a major complication, the presence of the $\left(\epsilon_{i / e}\right)^{-1}$ prefactor in front of the direct Coulomb term in Eq. (A6) requires that its contribution to the integral $\int d \mathbf{r} V(\mathbf{r}) \beta^{\prime}(\mathbf{r})$ must be truncated in two "inside/outside" integrals for a $\left\{\beta^{\prime}\right\}$ test orbital that spills out of the cavity. This is performed numerically using accurate but demanding quadrature rules (see supplementary material). ${ }^{83}$ As an important check, one verifies that the interaction energy between a Gaussian charge contained within the cavity with a charge significantly spilling out is completely symmetric whether the spilling orbital is considered as the source or the test orbital. The present formulation with explicit treatment of spilling charges is computationally more demanding than the original IEF-PCM formulation.

${ }^{1}$ J. Tomasi, B. Mennucci, and R. Cammi, Chem. Rev. 105, 2999 (2005).

${ }^{2}$ S. Miertuš, E. Scrocco, and J. Tomasi, Chem. Phys. 55, 117 (1981).

${ }^{3}$ D. M. York and M. Karplus, J. Phys. Chem. A 103, 11060 (1999).

${ }^{4}$ J. Pascual-Ahuir, E. Silla, and I. Tunon, J. Comput. Chem. 15, 1127 (1994).

${ }^{5}$ E. Cancès, B. Mennucci, and J. Tomasi, J. Chem. Phys. 107, 3032 (1997).

${ }^{6} \mathrm{E}$. Cancès and B. Mennucci, J. Math. Chem. 23, 309 (1998).

${ }^{7}$ D. M. Chipman, J. Chem. Phys. 110, 8012 (1999).

${ }^{8}$ D. M. Chipman, J. Chem. Phys. 124, 224111 (2006).

${ }^{9}$ I. Soteras, C. Curutchet, A. Bidon-Chanal, M. Orozco, and J. F. Luque, Mol. Struct.: THEOCHEM 727, 29 (2005).

${ }^{10}$ A. V. Marenich, C. J. Cramer, and D. G. Truhlar, J. Phys. Chem. B 113, 6378 (2009).

${ }^{11}$ A. Klamt and G. Schuurmann, J. Chem. Soc., Perkin Trans. 2 1993, 799

${ }^{12}$ D. Rinaldi and J.-L. Rivail, Theor. Chim. Acta 32, 57 (1973).

${ }^{13}$ D. Rinaldi, M. F. Ruiz-Lopez, and J.-L. Rivail, J. Chem. Phys. 78, 834 (1983).

${ }^{14}$ G. Scalmani and M. J. Frisch, J. Chem. Phys. 132, 114110 (2010).

${ }^{15}$ A. Klamt, Wiley Interdiscip. Rev.: Comput. Mol. Sci. 1, 699 (2011).

${ }^{16}$ B. Mennucci, Wiley Interdiscip. Rev.: Comput. Mol. Sci. 2, 386 (2012).

${ }^{17}$ P. C. Martin and J. Schwinger, Phys. Rev. 115, 1342 (1959).

${ }^{18}$ L. Hedin, Phys. Rev. 139, A796 (1965).

${ }^{19}$ G. Strinati, H. J. Mattausch, and W. Hanke, Phys. Rev. B 25, 2867 (1982).

${ }^{20}$ M. S. Hybertsen and S. G. Louie, Phys. Rev. B 34, 5390 (1986).

${ }^{21}$ R. W. Godby, M. Schlüter, and L. J. Sham, Phys. Rev. B 37, 10159 (1988).

${ }^{22}$ B. Farid, R. Daling, D. Lenstra, and W. van Haeringen, Phys. Rev. B 38, 7530 (1988).

${ }^{23}$ F. Aryasetiawan and O. Gunnarsson, Rep. Prog. Phys. 61, 237 (1998).

${ }^{24}$ B. Farid, Ground and Low-Lying Excited States of Interacting Electron Systems: A Survey and Some Critical Analyses, Electron Correlation in the Solid State, edited by N. H. March (Imperial College Press, 1999).

${ }^{25}$ G. Onida, L. Reining, and A. Rubio, Rev. Mod. Phys. 74, 601 (2002).

${ }^{26}$ M. L. Tiago and J. R. Chelikowsky, Solid State Commun. 136, 333 (2005).
${ }^{27}$ M. L. Tiago, P. R. C. Kent, R. Q. Hood, and F. A. Reboredo, J. Chem. Phys. 129, 084311 (2008).

${ }^{28}$ M. Palummo, C. Hogan, F. Sottile, P. Bagala, and A. Rubio, J. Chem. Phys. 131, 084102 (2009).

${ }^{29}$ P. Umari, G. Stenuit, and S. Baroni, Phys. Rev. B 81, 115104 (2010).

${ }^{30}$ X. Blase, C. Attaccalite, and V. Olevano, Phys. Rev. B 83, 115103 (2011).

${ }^{31}$ C. Faber, C. Attaccalite, V. Olevano, E. Runge, and X. Blase, Phys. Rev. B 83, 115123 (2011).

${ }^{32}$ X. Qian, P. Umari, and N. Marzari, Phys. Rev. B 84, 075103 (2011).

${ }^{33}$ D. Foerster, P. Koval, and D. Sánchez-Portal, J. Chem. Phys. 135, 074105 (2011).

${ }^{34}$ N. Marom, X. Ren, J. E. Moussa, J. R. Chelikowsky, and L. Kronik, Phys. Rev. B 84, 195143 (2011).

${ }^{35}$ C. Faber, J. L. Janssen, M. Côté, E. Runge, and X. Blase, Phys. Rev. B 84, 155104 (2011).

${ }^{36}$ B. Baumeier, D. Andrienko, and M. Rohlfing, J. Chem. Theory Comput. 8, 2790 (2012).

${ }^{37}$ N. Marom, F. Caruso, X. Ren, O. T. Hofmann, T. Körzdörfer, J. R. Chelikowsky, A. Rubio, M. Scheffler, and P. Rinke, Phys. Rev. B 86, 245127 (2012).

${ }^{38}$ S. Sharifzadeh, A. Biller, L. Kronik, and J. B. Neaton, Phys. Rev. B 85, 125307 (2012).

${ }^{39}$ F. Caruso, P. Rinke, X. Ren, M. Scheffler, and A. Rubio, Phys. Rev. B 86, 081102 (2012).

${ }^{40}$ P. Umari, L. Giacomazzi, F. De Angelis, M. Pastore, and S. Baroni, J. Chem. Phys. 139, 014709 (2013).

${ }^{41}$ F. Caruso, P. Rinke, X. Ren, A. Rubio, and M. Scheffler, Phys. Rev. B 88, 075105 (2013).

${ }^{42}$ T. A. Pham, H.-V. Nguyen, D. Rocca, and G. Galli, Phys. Rev. B 87, 155148 (2013).

${ }^{43}$ M. J. van Setten, F. Weigend, and F. Evers, J. Chem. Theory Comput. 9, 232 (2013).

${ }^{44}$ F. Bruneval and M. A. L. Marques, J. Chem. Theory Comput. 9, 324 (2013).

${ }^{45}$ B. Baumeier, M. Rohlfing, and D. Andrienko, J. Chem. Theory Comput. 10, 3104 (2014).

${ }^{46}$ P. Koval, D. Foerster, and D. Sánchez-Portal, Phys. Rev. B 89, 155417 (2014).

${ }^{47}$ H. Yin, Y. Ma, J. Mu, C. Liu, and M. Rohlfing, Phys. Rev. Lett. 112, 228301 (2014).

${ }^{48}$ J. Lischner, S. Sharifzadeh, J. Deslippe, J. B. Neaton, and S. G. Louie, Phys. Rev. B 90, 115130 (2014).

${ }^{49}$ F. Kaplan, F. Weigend, F. Evers, and M. J. van Setten, J. Chem. Theory Comput. 11, 5152 (2015).

${ }^{50}$ M. J. van Setten, F. Caruso, S. Sharifzadeh, X. Ren, M. Scheffler, F. Liu, J. Lischner, L. Lin, J. R. Deslippe, S. G. Louie, C. Yang, F. Weigend, J. B. Neaton, F. Evers, and P. Rinke, J. Chem. Theory Comput. 11, 5665 (2015).

${ }^{51}$ M. Govoni and G. Galli, J. Chem. Theory Comput. 11, 2680 (2015).

${ }^{52}$ J. Laflamme Janssen, B. Rousseau, and M. Côté, Phys. Rev. B 91, 125120 (2015).

${ }^{53}$ M. Kühn and F. Weigend, J. Chem. Theory Comput. 11, 969 (2015).

${ }^{54}$ J. W. Knight, X. Wang, L. Gallandi, O. Dolgounitcheva, X. Ren, J. V. Ortiz, P. Rinke, T. Körzdörfer, and N. Marom, J. Chem. Theory Comput. 12, 615 (2015).

${ }^{55}$ I. Duchemin and X. Blase, Phys. Rev. B 87, 245412 (2013).

${ }^{56}$ D. Niedzialek, I. Duchemin, T. B. de Queiroz, S. Osella, A. Rao, R. Friend, X. Blase, S. Kümmel, and D. Beljonne, Adv. Funct. Mater. 25, 1972 (2015).

${ }^{57}$ The Bethe-Salpeter equation (BSE) formalism tackles optical (neutral) excitations within the same Green's function framework and follows usually a $G W$ calculation. We will not address the issue of optical excitations in the present study.

${ }^{58}$ D. Jacquemin, I. Duchemin, and X. Blase, J. Chem. Theory Comput. 11, 3290 (2015).

${ }^{59}$ F. Bruneval, S. M. Hamed, and J. B. Neaton, J. Chem. Phys. 142, 244101 (2015).

${ }^{60}$ D. Jacquemin, I. Duchemin, and X. Blase, J. Chem. Theory Comput. 11, 5340 (2015).

${ }^{61}$ X. Blase and C. Attaccalite, Appl. Phys. Lett. 99, 171909 (2011).

${ }^{62}$ I. Duchemin, T. Deutsch, and X. Blase, Phys. Rev. Lett. 109, 167801 (2012).

${ }^{63}$ C. Faber, I. Duchemin, T. Deutsch, and X. Blase, Phys. Rev. B 86, 155315 (2012).

${ }^{64}$ C. Faber, P. Boulanger, I. Duchemin, C. Attaccalite, and X. Blase, J. Chem. Phys. 139, 194308 (2013).

${ }^{65}$ P. Boulanger, D. Jacquemin, I. Duchemin, and X. Blase, J. Chem. Theory Comput. 10, 1212 (2014). 
${ }^{66}$ M. Rohlfing, Phys. Rev. B 82, 205127 (2010).

${ }^{67}$ E. Coccia, D. Varsano, and L. Guidoni, J. Chem. Theory Comput. 10, 501 (2014).

${ }^{68}$ D. Varsano, E. Coccia, O. Pulci, A. M. Conte, and L. Guidoni, Comput. Theor. Chem. 1040-1041, 338 (2014).

${ }^{69}$ M. J. Frisch, G. W. Trucks, H. B. Schlegel, G. E. Scuseria, M. A. Robb, J. R. Cheeseman, G. Scalmani, V. Barone, B. Mennucci, G. A. Petersson, H. Nakatsuji, M. Caricato, X. Li, H. P. Hratchian, A. F. Izmaylov, J. Bloino, G. Zheng, J. L. Sonnenberg, M. Hada, M. Ehara, K. Toyota, R. Fukuda, J. Hasegawa, M. Ishida, T. Nakajima, Y. Honda, O. Kitao, H. Nakai, T. Vreven, J. A. Montgomery, Jr., J. E. Peralta, F. Ogliaro, M. Bearpark, J. J. Heyd, E. Brothers, K. N. Kudin, V. N. Staroverov, R. Kobayashi, J. Normand, K. Raghavachari, A. Rendell, J. C. Burant, S. S. Iyengar, J. Tomasi, M. Cossi, N. Rega, J. M. Millam, M. Klene, J. E. Knox, J. B. Cross, V. Bakken, C. Adamo, J. Jaramillo, R. Gomperts, R. E. Stratmann, O. Yazyev, A. J. Austin, R. Cammi, C. Pomelli, J. W. Ochterski, R. L. Martin, K. Morokuma, V. G. Zakrzewski, G. A. Voth, P. Salvador, J. J. Dannenberg, S. Dapprich, A. D. Daniels, O. Farkas, J. B. Foresman, J. V. Ortiz, J. Cioslowski, and D. J. Fox, Gaussian 09, Revision D.01, Gaussian, Inc., Wallingford, CT, 2009.

${ }^{70}$ E. Cancès, D. Gontier, and G. Stoltz, e-print arXiv:1506.01737.

${ }^{71}$ C. Adamo and V. Barone, J. Chem. Phys. 110, 6158 (1999).

${ }^{72}$ X. Ren, P. Rinke, V. Blum, J. Wieferink, A. Tkatchenko, A. Sanfilippo, K. Reuter, and M. Scheffler, New J. Phys. 14, 053020 (2012).

${ }^{73}$ H. Hayashi, N. Watanabe, Y. Udagawa, and C.-C. Kao, Proc. Natl. Acad. Sci. U. S. A. 97, 6264 (2000).

${ }^{74}$ J. B. Neaton, M. S. Hybertsen, and S. G. Louie, Phys. Rev. Lett. 97, 216405 (2006).

${ }^{75}$ J. M. Garcia-Lastra and K. S. Thygesen, Phys. Rev. Lett. 106, 187402 (2011).

${ }^{76}$ F. Bruneval, N. Vast, and L. Reining, Phys. Rev. B 74, 045102 (2006).

${ }^{77}$ M. Valiev, E. Bylaska, N. Govind, K. Kowalski, T. Straatsma, H. V. Dam, D. Wang, J. Nieplocha, E. Apra, T. Windus, and W. de Jong, Comput. Phys. Commun. 181, 1477 (2010).

${ }^{78}$ E. Pluhařová, P. Slaviček, and P. Jungwirth, Acc. Chem. Res. 48, 1209 (2015).

${ }^{79}$ A. Muñoz-Losa, D. Markovitsi, and R. Improta, Chem. Phys. Lett. 634, 20 (2015).
${ }^{80}$ P. Slavíček, B. Winter, M. Faubel, S. E. Bradforth, and P. Jungwirth, J. Am. Chem. Soc. 131, 6460 (2009).

${ }^{81}$ T. H. Dunning, J. Chem. Phys. 90, 1007 (1989).

${ }^{82}$ F. Weigend, A. Köhn, and C. Hättig, J. Chem. Phys. 116, 3175 (2002).

${ }^{83}$ See supplementary material at http://dx.doi.org/10.1063/1.4946778 for the analysis of the convergency (I) of the solvant induced shifts with respect to basis size and (II) of the reaction field matrix elements as a function of the number of surface tesserae and as compared to exact analytic results in the case of a perfect spherical surface. In (III) the quadrature rules employed to describe the "in" and "out" contributions to the energy of a basis orbital in a $v^{\text {reac }}$ reaction field, in the case of orbitals spilling out of the cavity, are described. Finally, a sketch of Equation (A4) (Appendix) is presented.

${ }^{84}$ M. Schreiber, M. R. Silva-Junior, S. P. A. Sauer, and W. Thiel, J. Chem. Phys. 128, 134110 (2008).

${ }^{85}$ Technically, the Gaussian09 non-equilibrium $\triangle \mathrm{SCF}$ calculations are performed saving the solvent parameters of the neutral form (noneq $=$ write ) and reading them in the next step devoted to the cationic form $($ noneq $=$ read $)$.

${ }^{86}$ A. Klamt, C. Moya, and J. Palomar, J. Chem. Theory Comput. 11, 4220 (2015).

${ }^{87}$ V. Barone and M. Cossi, J. Phys. Chem. A 102, 1995 (1998).

${ }^{88}$ A. Rappé, C. Casewit, K. Colwell, W. G. III, and W. Skiff, J. Am. Chem. Soc. 114, 10024 (1992).

${ }^{89}$ Due to the ZPE contribution to experimental vertical data, not present in the theoretical calculations, one should expect as a matter of fact that the theoretical value stands above the experimental range.

${ }^{90}$ D. Dougherty, E. Younathan, R. Voll, S. Abdulnur, and S. McGlynn, J. Electron Spectrosc. Relat. Phenom. 13, 379 (1978).

${ }^{91}$ D. Roca-Sanjuán, M. Rubio, M. Merchán, and L. Serrano-Andrés, J. Chem. Phys. 125, 084302 (2006).

${ }^{92}$ D. Roca-Sanjuán, M. Merchán, L. Serrano-Andrés, and M. Rubio, J. Chem. Phys. 129, 095104 (2008).

${ }^{93}$ A. Trofimov, J. Schirmer, V. Kobychev, A. Potts, D. Holland, and L. Karlsson, J. Phys. B: At., Mol. Opt. Phys. 39, 305 (2006).

${ }^{94}$ G. D' Avino, L. Muccioli, C. Zannoni, D. Beljonne, and Z. G. Soos, J. Chem. Theory Comput. 10, 4959 (2014).

${ }^{95} \mathrm{~W}$. Hackbusch, Integral Equations-Theory and Numerical Treatment (Birkhäuser, Basel, 1995), Vol. 120. 